Journal of Scientific Perspectives

Volume 5, Issue 1, Year 2021, pp. 1-23

E - ISSN: 2587-3008

URL: https://journals.gen.tr/jsp

DOİ: https://doi.org/10.26900/jsp.5.1.02

Research Article

\title{
A Study On the Sums of Squares of Generalized Tribonacci Numbers: Closed Form Formulas of $\sum_{k=0}^{n} k x^{k} W_{k}^{2}$
}

\author{
Yüksel SOYKAN \\ Department of Mathematics, \\ Art and Science Faculty, Zonguldak Bülent Ecevit University, \\ 67100, Zonguldak, TURKEY \\ e-mail: yuksel_soykan@hotmail.com \\ ORCID ID: https://orcid.org/0000-0002-1895-211X
}

Received: 19 August 2020, Accepted: 28 November 2020

\begin{abstract}
In this paper, closed forms of the sum formulas $\sum_{k=0}^{n} k x^{k} W_{k}^{2}, \sum_{k=0}^{n} k x^{k} W_{k+2} W_{k}$ and $\sum_{k=0}^{n} k x^{k} W_{k+1} W_{k}$ for the squares of generalized Tribonacci numbers are presented. Here, $\left\{W_{m}\right\}_{m \in \mathbb{Z}}$ is the generalized Tribonacci sequence, $n$ is a non-negative integer and $x$ is a real or complex number. As special cases, we give summation formulas of Tribonacci, Tribonacci-Lucas, Padovan, Perrin numbers and the other third order recurrence relations.
\end{abstract}

2020 Mathematics Subject Classification. 11B39, 11B83.

Keywords. Sums of squares, third order recurrence, generalized Tribonacci numbers, Padovan numbers, Perrin numbers, Narayana numbers.

\section{Introduction}

The generalized Tribonacci sequence $\left\{W_{n}\left(W_{0}, W_{1}, W_{2} ; r, s, t\right)\right\}_{n \geq 0}$ (or shortly $\left.\left\{W_{n}\right\}_{n \geq 0}\right)$ is defined as follows:

$$
W_{n}=r W_{n-1}+s W_{n-2}+t W_{n-3}, \quad W_{0}=a, W_{1}=b, W_{2}=c, \quad n \geq 3
$$

where $W_{0}, W_{1}, W_{2}$ are arbitrary complex numbers and $r, s, t$ are real numbers. The generalized Tribonacci sequence has been studied by many authors, see for example $[1,2,6,7,13,14,19,20,21,23,35,36,37,38]$.

The sequence $\left\{W_{n}\right\}_{n \geq 0}$ can be extended to negative subscripts by defining

$$
W_{-n}=-\frac{s}{t} W_{-(n-1)}-\frac{r}{t} W_{-(n-2)}+\frac{1}{t} W_{-(n-3)}
$$

for $n=1,2,3, \ldots$ when $t \neq 0$. Therefore, recurrence (1.1) holds for all integer $n$.

In literature, for example, the following names and notations (see Table 1) are used for the special case of $r, s, t$ and initial values. 
Table 1 A few special case of generalized Tribonacci sequences.

\begin{tabular}{|c|c|c|}
\hline Sequences (Numbers) & Notation & OEIS $[22]$ \\
\hline Tribonacci & $\left\{T_{n}\right\}=\left\{V_{n}(0,1,1 ; 1,1,1)\right\}$ & $\mathrm{A} 000073, \mathrm{~A} 057597$ \\
\hline Tribonacci-Lucas & $\left\{K_{n}\right\}=\left\{V_{n}(3,1,3 ; 1,1,1)\right\}$ & $\mathrm{A} 001644, \mathrm{~A} 073145$ \\
\hline third order Pell & $\left\{P_{n}^{(3)}\right\}=\left\{V_{n}(0,1,2 ; 2,1,1)\right\}$ & A 077939, A077978 \\
\hline third order Pell-Lucas & $\left\{Q_{n}^{(3)}\right\}=\left\{V_{n}(3,2,6 ; 2,1,1)\right\}$ & A 276225, A 276228 \\
\hline third order modified Pell & $\left\{E_{n}^{(3)}\right\}=\left\{V_{n}(0,1,1 ; 2,1,1)\right\}$ & A077997, A078049 \\
\hline Padovan (Cordonnier) & $\left\{P_{n}\right\}=\left\{V_{n}(1,1,1 ; 0,1,1)\right\}$ & A000931 \\
\hline Perrin (Padovan-Lucas) & $\left\{E_{n}\right\}=\left\{V_{n}(3,0,2 ; 0,1,1)\right\}$ & A 001608, A078712 \\
\hline Padovan-Perrin & $\left\{S_{n}\right\}=\left\{V_{n}(0,0,1 ; 0,1,1)\right\}$ & A000931, A176971 \\
\hline Pell-Padovan & $\left\{R_{n}\right\}=\left\{V_{n}(1,1,1 ; 0,2,1)\right\}$ & A 066983, A128587 \\
\hline Pell-Perrin & $\left\{C_{n}\right\}=\left\{V_{n}(3,0,2 ; 0,2,1)\right\}$ & \\
\hline Jacobsthal-Padovan & $\left\{Q_{n}\right\}=\left\{V_{n}(1,1,1 ; 0,1,2)\right\}$ & A159284 \\
\hline Jacobsthal-Perrin (-Lucas) & $\left\{L_{n}\right\}=\left\{V_{n}(3,0,2 ; 0,1,2)\right\}$ & A072328 \\
\hline Narayana & $\left\{N_{n}\right\}=\left\{V_{n}(0,1,1 ; 1,0,1)\right\}$ & A078012 \\
\hline Narayana-Lucas & $\left\{U_{n}\right\}=\left\{V_{n}(3,1,1 ; 1,0,1)\right\}$ & A001609 \\
\hline Narayana-Perrin & $\left\{H_{n}\right\}=\left\{V_{n}(3,0,2 ; 1,0,1)\right\}$ & \\
\hline third order Jacobsthal & $\left\{J_{n}^{(3)}\right\}=\left\{V_{n}(0,1,1 ; 1,1,2)\right\}$ & A 077947 \\
\hline third order Jacobsthal-Lucas & $\left\{j_{n}^{(3)}\right\}=\left\{V_{n}(2,1,5 ; 1,1,2)\right\}$ & A 226308 \\
\hline 3 -primes & $\left\{G_{n}\right\}=\left\{V_{n}(0,1,2 ; 2,3,5)\right\}$ & \\
\hline Lucas 3-primes & $\left\{H_{n}\right\}=\left\{V_{n}(3,2,10 ; 2,3,5)\right\}$ & \\
\hline modified 3-primes & $\left\{E_{n}\right\}=\left\{V_{n}(0,1,1 ; 2,3,5)\right\}$ & \\
\hline
\end{tabular}

evaluation of sums of powers of these sequences is a challenging issue. Two pretty examples are

$\sum_{k=0}^{n} k(-1)^{k} T_{k}^{2}=\frac{1}{4}\left((-1)^{n}\left((n+1) T_{n+3}^{2}-(2 n+1) T_{n+2}^{2}+(3 n+2) T_{n+1}^{2}-2(n+2) T_{n+1} T_{n+3}+2 T_{n+2} T_{n+1}\right)+1\right)$

and

$\sum_{k=0}^{n} k(-1)^{k} N_{k}^{2}=\frac{1}{9}\left((-1)^{n}\left((3 n+7) N_{n+3}^{2}-(6 n+5) N_{n+2}^{2}+(6 n-1) N_{n+1}^{2}-6 N_{n+3} N_{n+2}-2(3 n+7) N_{n+3} N_{n+1}+\right.\right.$ $\left.\left.2(3 n+10) N_{n+2} N_{n+1}\right)-1\right)$.

In this work, we derive expressions for sums of second powers of generalized Tribonacci numbers. We present some works on sum formulas of powers of the numbers in the following Table 2.

Table 2. A few special study on sum formulas of second, third and arbitrary powers.

\begin{tabular}{cccc}
\hline Name of sequence & sums of second powers & sums of third powers & sums of powers \\
\hline Generalized Fibonacci & {$[3,4,10,11,12,24,33,30]$} & {$[9,25,27,28,31,32,39]$} & {$[5,8,15]$} \\
Generalized Tribonacci & {$[17,26,29]$} & & \\
Generalized Tetranacci & {$[16,18,34]$} & & \\
\hline Let
\end{tabular}

$$
\Delta=\left(-t^{2} x^{3}+s x+r t x^{2}+1\right)\left(r^{2} x-s^{2} x^{2}+t^{2} x^{3}+2 s x+2 r t x^{2}-1\right) .
$$

THEOREM 1.1. If $\Delta \neq 0$ then

(a):

$$
\sum_{k=0}^{n} x^{k} W_{k}^{2}=\frac{\Delta_{1}}{\Delta}
$$


(b):

$$
\sum_{k=0}^{n} x^{k} W_{k+1} W_{k}=\frac{\Delta_{2}}{\Delta}
$$

(c):

$$
\sum_{k=0}^{n} x^{k} W_{k+2} W_{k}=\frac{\Delta_{3}}{\Delta}
$$

where

$\Delta_{1}=-x^{n+3}\left(t^{2} x^{3}+s x+r t x^{2}-1\right) W_{n+3}^{2}-x^{n+2}\left(r^{2} x+t^{2} x^{3}+s x+r^{2} t^{2} x^{4}+r t x^{2}+r^{2} s x^{2}+r^{3} t x^{3}+2 r s t x^{3}-1\right)$ $W_{n+2}^{2}-x^{n+1}\left(r^{2} x+s^{2} x^{2}-s^{3} x^{3}+t^{2} x^{3}+s x+r^{2} t^{2} x^{4}+s^{2} t^{2} x^{5}+r t x^{2}+r^{2} s x^{2}+r^{3} t x^{3}+4 r s t x^{3}-r s^{2} t x^{4}-1\right)$ $W_{n+1}^{2}+x^{2}\left(t^{2} x^{3}+s x+r t x^{2}-1\right) W_{2}^{2}+x\left(r^{2} x+t^{2} x^{3}+s x+r^{2} t^{2} x^{4}+r t x^{2}+r^{2} s x^{2}+r^{3} t x^{3}+2 r s t x^{3}-1\right)$ $W_{1}^{2}+\left(r^{2} x+s^{2} x^{2}-s^{3} x^{3}+t^{2} x^{3}+s x+r^{2} t^{2} x^{4}+s^{2} t^{2} x^{5}+r t x^{2}+r^{2} s x^{2}+r^{3} t x^{3}+4 r s t x^{3}-r s^{2} t x^{4}-1\right)$ $W_{0}^{2}+2 x^{n+4}(r+t x)(s+r t x) W_{n+3} W_{n+2}+2 x^{n+4} t\left(r+s t x^{2}\right) W_{n+3} W_{n+1}-2 x^{n+4} t(s x-1)(s+r t x) W_{n+2} W_{n+1}-$ $2 x^{3}(r+t x)(s+r t x) W_{2} W_{1}-2 t x^{3}\left(r+s t x^{2}\right) W_{2} W_{0}+2 x^{3} t(s x-1)(s+r t x) W_{1} W_{0}$

and

$\Delta_{2}=x^{n+3}\left(r+s t x^{2}\right) W_{n+3}^{2}+x^{n+4}(t+r s)(s+r t x) W_{n+2}^{2}+x^{n+4} t^{2}\left(r+s t x^{2}\right) W_{n+1}^{2}-x^{n+2}\left(r^{2} x+s^{2} x^{2}+\right.$ $\left.t^{2} x^{3}+2 r s t x^{3}-1\right) W_{n+3} W_{n+2}+x^{n+3} t\left(r^{2} x-s^{2} x^{2}-t^{2} x^{3}+1\right) W_{n+3} W_{n+1}-x^{n+1}\left(r^{2} x+s^{2} x^{2}-s^{3} x^{3}+t^{2} x^{3}+\right.$ $\left.s x+r t x^{2}+r^{2} s x^{2}+r^{3} t x^{3}-r t^{3} x^{5}-s t^{2} x^{4}+2 r s t x^{3}-r s^{2} t x^{4}-1\right) W_{n+2} W_{n+1}-x^{2}\left(r+s t x^{2}\right) W_{2}^{2}-x^{3}(t+$ $r s)(s+r t x) W_{1}^{2}-x^{3} t^{2}\left(r+s t x^{2}\right) W_{0}^{2}+x\left(r^{2} x+s^{2} x^{2}+t^{2} x^{3}+2 r s t x^{3}-1\right) W_{2} W_{1}-x^{2} t\left(r^{2} x-s^{2} x^{2}-t^{2} x^{3}+\right.$ 1) $W_{2} W_{0}+\left(r^{2} x+s^{2} x^{2}-s^{3} x^{3}+t^{2} x^{3}+s x+r t x^{2}+r^{2} s x^{2}+r^{3} t x^{3}-r t^{3} x^{5}-s t^{2} x^{4}+2 r s t x^{3}-r s^{2} t x^{4}-1\right)$ $W_{1} W_{0}$

and

$\Delta_{3}=x^{n+3}\left(s-s^{2} x+r^{2}+r t x\right) W_{n+3}^{2}+x^{n+2}\left(s-s^{2} x+r^{2} t^{2} x^{3}-r^{2} s x+r t^{3} x^{4}-r s^{2} t x^{3}\right) W_{n+2}^{2}+x^{n+4} t^{2}\left(s-s^{2} x+\right.$ $\left.r^{2}+r t x\right) W_{n+1}^{2}-x^{n+2}(r+t x)\left(r^{2} x-s^{2} x^{2}+t^{2} x^{3}-1\right) W_{n+3} W_{n+2}-x^{n+1}\left(r^{2} x+s^{2} x^{2}-s^{3} x^{3}+t^{2} x^{3}+s x+r^{2} s x^{2}-\right.$ $\left.s t^{2} x^{4}+2 r s t x^{3}-1\right) W_{n+3} W_{n+1}+x^{n+2} t(s x-1)\left(r^{2} x-s^{2} x^{2}+t^{2} x^{3}-1\right) W_{n+2} W_{n+1}-x^{2}\left(s-s^{2} x+r^{2}+r t x\right) W_{2}^{2}+$ $x\left(-s+s^{2} x-r^{2} t^{2} x^{3}+r^{2} s x-r t^{3} x^{4}+r s^{2} t x^{3}\right) W_{1}^{2}-x^{3} t^{2}\left(s-s^{2} x+r^{2}+r t x\right) W_{0}^{2}+x(r+t x)\left(r^{2} x-s^{2} x^{2}+t^{2} x^{3}-\right.$ 1) $W_{2} W_{1}+\left(r^{2} x+s^{2} x^{2}-s^{3} x^{3}+t^{2} x^{3}+s x+r^{2} s x^{2}-s t^{2} x^{4}+2 r s t x^{3}-1\right) W_{2} W_{0}-x t(s x-1)\left(r^{2} x-s^{2} x^{2}+t^{2} x^{3}-1\right)$ $W_{1} W_{0}$.

Proof. The proof is given in [29, Theorem 3.1.].

\section{Main Result}

Let

$$
\Omega=\left(-t^{2} x^{3}+s x+r t x^{2}+1\right)^{2}\left(r^{2} x-s^{2} x^{2}+t^{2} x^{3}+2 s x+2 r t x^{2}-1\right)^{2}
$$

Theorem 2.1. Let $x$ be a real or complex number. If $\Omega \neq 0$ then

(a):

$$
\sum_{k=0}^{n} k x^{k} W_{k}^{2}=\frac{\Omega_{1}}{\Omega}
$$

(b):

$$
\sum_{k=0}^{n} k x^{k} W_{k+1} W_{k}=\frac{\Omega_{2}}{\Omega}
$$


(c):

$$
\sum_{k=0}^{n} k x^{k} W_{k+2} W_{k}=\frac{\Omega_{3}}{\Omega}
$$

where

$$
\Omega_{1}=\sum_{k=1}^{12} \Gamma_{k}, \quad \Omega_{2}=\sum_{k=1}^{12} \Phi_{k}, \quad \Omega_{3}=\sum_{k=1}^{12} \Psi_{k}
$$

with

$\Gamma_{1}=x^{n+3}\left(n\left(t^{2} x^{3}-s x-r t x^{2}-1\right)\left(t^{2} x^{3}+s x+r t x^{2}-1\right)\left(r^{2} x-s^{2} x^{2}+t^{2} x^{3}+2 s x+2 r t x^{2}-1\right)+2 r^{2} x-\right.$ $2 s^{2} x^{2}-2 s^{3} x^{3}+6 t^{2} x^{3}+s^{4} x^{4}-3 t^{4} x^{6}+6 s x-2 r^{2} s^{2} x^{3}-9 r^{2} t^{2} x^{4}-2 r^{4} t^{2} x^{5}-6 s^{2} t^{2} x^{5}-4 r^{3} t^{3} x^{6}-2 r^{2} t^{4} x^{7}+4 s^{3} t^{2}$ $x^{6}-s^{2} t^{4} x^{8}+6 r t x^{2}-6 r t^{3} x^{5}-6 s t^{2} x^{4}-12 r^{2} s t^{2} x^{5}+2 r s^{2} t^{3} x^{7}-6 r s^{2} t x^{4}-4 r^{3} s t x^{4}+2 r s^{3} t x^{5}-12 r s t^{3} x^{6}-$ $\left.3-2 r^{2} s x^{2}-6 r s t x^{3}+r^{2} s^{2} t^{2} x^{6}-4 r^{3} t x^{3}\right) W_{n+3}^{2}$,

$\Gamma_{2}=x^{n+2}\left(n\left(r^{2} x+t^{2} x^{3}+s x+r^{2} t^{2} x^{4}+r t x^{2}+r^{2} s x^{2}+r^{3} t x^{3}+2 r s t x^{3}-1\right)\left(t^{2} x^{3}-s x-r t x^{2}-1\right)\left(r^{2} x-\right.\right.$ $\left.s^{2} x^{2}+t^{2} x^{3}+2 s x+2 r t x^{2}-1\right)+4 r^{2} x-2 r^{4} x^{2}-2 s^{2} x^{2}+3 t^{2} x^{3}-t^{6} x^{9}+4 s x-5 r^{2} s^{2} x^{3}-2 r^{2} s^{3} x^{4}-2 r^{2} t^{2} x^{4}-2 r^{4}$ $s^{2} x^{4}+r^{2} s^{4} x^{5}-8 r^{4} t^{2} x^{5}-7 s^{2} t^{2} x^{5}-12 r^{3} t^{3} x^{6}-11 r^{2} t^{4} x^{7}+4 s^{3} t^{2} x^{6}-2 r^{6} t^{2} x^{6}-4 r^{5} t^{3} x^{7}-2 r^{4} t^{4} x^{8}-4 r^{4} s$ $x^{3}-2 r t^{3} x^{5}-2 s t^{2} x^{4}-4 r^{5} t x^{4}-2 r t^{5} x^{8}-2 s t^{4} x^{7}-20 r^{2} s t^{2} x^{5}-14 r^{3} s^{2} t x^{5}+4 r s^{2} t^{3} x^{7}+2 r^{3} s^{3} t x^{6}-16 r^{4} s t^{2} x^{6}-$ $14 r^{3} s t^{3} x^{7}+2 r s t x^{3}-18 r^{2} s^{2} t^{2} x^{6}+6 r^{2} s^{3} t^{2} x^{7}+r^{4} s^{2} t^{2} x^{7}+2 r^{3} s^{2} t^{3} x^{8}-r^{2} s^{2} t^{4} x^{9}-8 r s^{2} t x^{4}-16 r^{3} s t x^{4}-6$ $\left.r s^{3} t x^{5}-18 r s t^{3} x^{6}+4 r s^{4} t x^{6}-4 r^{5} s t x^{5}-2 r s t^{5} x^{9}-2+4 r t x^{2}\right) W_{n+2}^{2}$,

$\Gamma_{3}=x^{n+1}\left(n\left(t^{2} x^{3}-s x-r t x^{2}-1\right)\left(r^{2} x+s^{2} x^{2}-s^{3} x^{3}+t^{2} x^{3}+s x+r^{2} t^{2} x^{4}+s^{2} t^{2} x^{5}+r t x^{2}+r^{2} s x^{2}+r^{3} t x^{3}+\right.\right.$ $\left.4 r s t x^{3}-r s^{2} t x^{4}-1\right)\left(r^{2} x-s^{2} x^{2}+t^{2} x^{3}+2 s x+2 r t x^{2}-1\right)+2 r^{2} x-r^{4} x^{2}+s^{2} x^{2}-4 s^{3} x^{3}+s^{4} x^{4}+2 s^{5} x^{5}-s^{6} x^{6}+$ $3 t^{4} x^{6}-2 t^{6} x^{9}+2 s x-4 r^{2} s^{2} x^{3}-r^{4} s^{2} x^{4}+2 r^{2} s^{4} x^{5}-4 r^{4} t^{2} x^{5}-2 s^{2} t^{2} x^{5}-8 r^{3} t^{3} x^{6}-10 r^{2} t^{4} x^{7}-r^{6} t^{2} x^{6}-2 r^{5} t^{3} x^{7}-$ $2 s^{4} t^{2} x^{7}-2 r^{4} t^{4} x^{8}-8 s^{2} t^{4} x^{8}-2 r^{3} t^{5} x^{9}+2 s^{5} t^{2} x^{8}-r^{2} t^{6} x^{10}+4 s^{3} t^{4} x^{9}-s^{4} t^{4} x^{10}+2 r t^{3} x^{5}+2 s t^{2} x^{4}-2 r^{5} t$ $x^{4}-4 r t^{5} x^{8}-4 s t^{4} x^{7}-14 r^{2} s t^{2} x^{5}-8 r^{3} s^{2} t x^{5}+4 r^{3} s^{3} t x^{6}-10 r^{4} s t^{2} x^{6}-10 r^{3} s t^{3} x^{7}-8 r s^{3} t^{3} x^{8}-6 r^{2} s t^{4} x^{8}+$ $2 r s^{4} t^{3} x^{9}+2 r s^{2} t^{5} x^{10}+6 r s t x^{3}-19 r^{2} s^{2} t^{2} x^{6}+8 r^{2} s^{3} t^{2} x^{7}+2 r^{4} s^{2} t^{2} x^{7}-r^{2} s^{4} t^{2} x^{8}-2 r^{2} s^{2} t^{4} x^{9}-12 r s^{2} t x^{4}-$ $\left.12 r^{3} s t x^{4}-4 r s^{3} t x^{5}-16 r s t^{3} x^{6}+10 r s^{4} t x^{6}-2 r^{5} s t x^{5}-2 r s^{5} t x^{7}-8 r s t^{5} x^{9}-1+2 r t x^{2}-2 r^{4} s x^{3}\right) W_{n+1}^{2}$,

$\Gamma_{4}=2 x^{n+4}\left(n(r+t x)\left(-t^{2} x^{3}+s x+r t x^{2}+1\right)(s+r t x)\left(r^{2} x-s^{2} x^{2}+t^{2} x^{3}+2 s x+2 r t x^{2}-1\right)+2 r^{3} s^{2} x^{2}-\right.$ $4 r s+8 r^{3} t^{2} x^{3}+8 r^{2} t^{3} x^{4}+2 r^{5} t^{2} x^{4}+4 r^{4} t^{3} x^{5}-s^{2} t^{3} x^{5}+2 r^{3} t^{4} x^{6}+3 r s^{2} x+3 r^{3} s x-5 r^{2} t x+2 r s^{3} x^{2}-6 r t^{2} x^{2}-r s^{4}$ $x^{3}+4 r^{4} t x^{2}+4 s^{2} t x^{2}+3 s^{3} t x^{3}+6 r t^{4} x^{5}+4 s t^{3} x^{4}-2 s^{4} t x^{4}+s t^{5} x^{7}-5 s t x+10 r^{2} s^{2} t x^{3}+12 r s^{2} t^{2} x^{4}-2 r^{2} s^{3} t x^{4}+$ $\left.14 r^{3} s t^{2} x^{4}-5 r s^{3} t^{2} x^{5}+13 r^{2} s t^{3} x^{5}+r s^{2} t^{4} x^{7}-r^{3} s^{2} t^{2} x^{5}-2 r^{2} s^{2} t^{3} x^{6}+10 r^{2} s t x^{2}+10 r s t^{2} x^{3}+4 r^{4} s t x^{3}\right) W_{n+3} W_{n+2}$,

$\Gamma_{5}=2 t x^{n+4}\left(n\left(-t^{2} x^{3}+s x+r t x^{2}+1\right)\left(r+s t x^{2}\right)\left(r^{2} x-s^{2} x^{2}+t^{2} x^{3}+2 s x+2 r t x^{2}-1\right)+3 r^{3} x-\right.$ $4 r+r^{2} t^{3} x^{5}-2 s^{2} t^{3} x^{6}+s^{3} t^{3} x^{7}-6 s t x^{2}+2 r s^{2} x^{2}+2 r^{3} s x^{2}-r s^{3} x^{3}+2 r^{2} t x^{2}+2 r t^{2} x^{3}+r^{4} t x^{3}+5 s^{2} t x^{3}+4$ $s^{3} t x^{4}+2 r t^{4} x^{6}+6 s t^{3} x^{5}-3 s^{4} t x^{5}+3 r s x+11 r s^{2} t^{2} x^{5}+3 r^{3} s t^{2} x^{5}-2 r s^{3} t^{2} x^{6}+2 r^{2} s t^{3} x^{6}+9 r^{2} s t x^{3}+4 r s t^{2} x^{4}-r s t^{4}$ $\left.x^{7}+4 r^{2} s^{2} t x^{4}\right) W_{n+3} W_{n+1}$,

$\Gamma_{6}=2 t x^{n+4}\left(n(s x-1)(s+r t x)\left(t^{2} x^{3}-s x-r t x^{2}-1\right)\left(r^{2} x-s^{2} x^{2}+t^{2} x^{3}+2 s x+2 r t x^{2}-1\right)+8 s^{2} x-4 s-\right.$ $2 s^{3} x^{2}-4 s^{4} x^{3}+2 s^{5} x^{4}-2 r^{2} s^{2} x^{2}-3 r^{2} s^{3} x^{3}+3 r^{2} t^{2} x^{3}+2 r^{4} t^{2} x^{4}-4 s^{2} t^{2} x^{4}+r^{3} t^{3} x^{5}-s^{2} t^{4} x^{7}+4 r^{3} t x^{2}+4 r t^{3} x^{4}+2$ $s t^{2} x^{3}+r t^{5} x^{7}+2 s t^{4} x^{6}-5 r t x+4 r^{2} s t^{2} x^{4}-6 r^{3} s^{2} t x^{4}+2 r s^{2} t^{3} x^{6}-3 r^{4} s t^{2} x^{5}-2 r^{3} s t^{3} x^{6}-r s^{3} t^{3} x^{7}+r^{2} s t^{4} x^{7}+$ $\left.12 r s t x^{2}-14 r^{2} s^{2} t^{2} x^{5}+2 r^{2} s^{3} t^{2} x^{6}-r s^{2} t x^{3}-r^{3} s t x^{3}-14 r s^{3} t x^{4}-6 r s t^{3} x^{5}+4 r s^{4} t x^{5}+3 r^{2} s x\right) W_{n+2} W_{n+1}$,

$\Gamma_{7}=x^{2}\left(-r^{2} x+2 s^{2} x^{2}-3 t^{2} x^{3}+t^{6} x^{9}-4 s x+r^{2} s^{2} x^{3}+8 r^{2} t^{2} x^{4}+r^{4} t^{2} x^{5}+7 s^{2} t^{2} x^{5}+2 r^{3} t^{3} x^{6}+2 r^{2} t^{4} x^{7}-\right.$ $4 s^{3} t^{2} x^{6}-4 r t x^{2}+2 r^{2} s x^{2}+4 r^{3} t x^{3}+2 r t^{3} x^{5}+2 s t^{2} x^{4}+2 r t^{5} x^{8}+2 s t^{4} x^{7}+6 r^{2} s t^{2} x^{5}-2 r s^{2} t^{3} x^{7}+8 r s t x^{3}+$ $\left.2 r^{3} s t x^{4}+10 r s t^{3} x^{6}+2\right) W_{2}^{2}$, 
$\Gamma_{8}=x\left(-2 r^{2} x+r^{4} x^{2}+2 s^{2} x^{2}-2 s^{3} x^{3}+s^{4} x^{4}-3 t^{4} x^{6}+2 t^{6} x^{9}-2 s x+2 r^{2} s^{2} x^{3}+2 r^{2} s^{3} x^{4}+r^{4} s^{2} x^{4}+\right.$ $4 r^{4} t^{2} x^{5}+8 s^{2} t^{2} x^{5}+8 r^{3} t^{3} x^{6}+10 r^{2} t^{4} x^{7}-4 s^{3} t^{2} x^{6}+r^{6} t^{2} x^{6}+2 r^{5} t^{3} x^{7}+2 r^{4} t^{4} x^{8}-s^{2} t^{4} x^{8}+2 r^{3} t^{5} x^{9}+r^{2} t^{6} x^{10}-$ $2 r t x^{2}+2 r^{4} s x^{3}-2 r t^{3} x^{5}-2 s t^{2} x^{4}+2 r^{5} t x^{4}+4 r t^{5} x^{8}+4 s t^{4} x^{7}+10 r^{2} s t^{2} x^{5}+8 r^{3} s^{2} t x^{5}-2 r s^{2} t^{3} x^{7}+8 r^{4} s t^{2} x^{6}+$ $10 r^{3} s t^{3} x^{7}-2 r s^{3} t^{3} x^{8}+4 r^{2} s t^{4} x^{8}+2 r s t x^{3}+10 r^{2} s^{2} t^{2} x^{6}-4 r^{2} s^{3} t^{2} x^{7}-2 r^{3} s^{2} t^{3} x^{8}+6 r^{3} s t x^{4}+6 r s^{3} t x^{5}+12 r s t^{3} x^{6}-$ $\left.2 r s^{4} t x^{6}+2 r^{5} s t x^{5}+4 r s t^{5} x^{9}+1\right) W_{1}^{2}$,

$\Gamma_{9}=t^{2} x^{3}\left(-2 r^{2} x+2 s^{2} x^{2}+2 s^{3} x^{3}-6 t^{2} x^{3}-s^{4} x^{4}+3 t^{4} x^{6}-6 s x+2 r^{2} s^{2} x^{3}+9 r^{2} t^{2} x^{4}+2 r^{4} t^{2} x^{5}+6 s^{2} t^{2} x^{5}+\right.$ $4 r^{3} t^{3} x^{6}+2 r^{2} t^{4} x^{7}-4 s^{3} t^{2} x^{6}+s^{2} t^{4} x^{8}-6 r t x^{2}+2 r^{2} s x^{2}+4 r^{3} t x^{3}+6 r t^{3} x^{5}+6 s t^{2} x^{4}+12 r^{2} s t^{2} x^{5}-2 r s^{2} t^{3} x^{7}+$ $\left.6 r s t x^{3}-r^{2} s^{2} t^{2} x^{6}+6 r s^{2} t x^{4}+4 r^{3} s t x^{4}-2 r s^{3} t x^{5}+12 r s t^{3} x^{6}+3\right) W_{0}^{2}$,

$\Gamma_{10}=-2 x^{3}\left(-3 r s+r^{3} s^{2} x^{2}+6 r^{3} t^{2} x^{3}+5 r^{2} t^{3} x^{4}+r^{5} t^{2} x^{4}+2 r^{4} t^{3} x^{5}+2 r^{3} t^{4} x^{6}+2 r^{2} t^{5} x^{7}-s^{3} t^{3} x^{6}+2 r s^{2} x+\right.$ $2 r^{3} s x-4 r^{2} t x+r s^{3} x^{2}-5 r t^{2} x^{2}+3 r^{4} t x^{2}+3 s^{2} t x^{2}+2 s^{3} t x^{3}+4 r t^{4} x^{5}+2 s t^{3} x^{4}-s^{4} t x^{4}+r t^{6} x^{8}+2 s t^{5} x^{7}-4 s t x+$ $\left.4 r^{2} s^{2} t x^{3}+8 r s^{2} t^{2} x^{4}+7 r^{3} s t^{2} x^{4}-4 r s^{3} t^{2} x^{5}+10 r^{2} s t^{3} x^{5}-2 r^{2} s^{2} t^{3} x^{6}+7 r^{2} s t x^{2}+6 r s t^{2} x^{3}+2 r^{4} s t x^{3}+3 r s t^{4} x^{6}\right)$ $W_{2} W_{1}$

$\Gamma_{11}=-2 t x^{3}\left(-3 r+2 r^{3} x-r^{3} t^{2} x^{4}+2 r^{2} t^{3} x^{5}-s^{2} t^{3} x^{6}-5 s t x^{2}+r s^{2} x^{2}+r^{3} s x^{2}+r^{2} t x^{2}+4 s^{2} t x^{3}+3 s^{3} t x^{4}+\right.$ $\left.3 r t^{4} x^{6}+4 s t^{3} x^{5}-2 s^{4} t x^{5}+s t^{5} x^{8}+2 r s x+4 r^{2} s^{2} t x^{4}+6 r s^{2} t^{2} x^{5}+2 r^{3} s t^{2} x^{5}-r s^{3} t^{2} x^{6}+r^{2} s t^{3} x^{6}+4 r^{2} s t x^{3}+4 r s t^{2} x^{4}\right)$ $W_{2} W_{0}$

$\Gamma_{12}=2 t x^{3}\left(3 s-6 s^{2} x+2 s^{3} x^{2}+2 s^{4} x^{3}-s^{5} x^{4}+2 r^{2} s^{2} x^{2}+2 r^{2} s^{3} x^{3}-2 r^{2} t^{2} x^{3}-r^{4} t^{2} x^{4}+s^{2} t^{2} x^{4}-r^{2} t^{4} x^{6}+\right.$ $2 s^{3} t^{2} x^{5}-s^{4} t^{2} x^{6}+2 s^{2} t^{4} x^{7}-2 r^{2} s x-3 r^{3} t x^{2}-2 r t^{3} x^{4}-2 r t^{5} x^{7}-3 s t^{4} x^{6}+4 r t x+4 r^{3} s^{2} t x^{4}+r s^{2} t^{3} x^{6}+2 r^{4} s t^{2} x^{5}+$ $\left.r^{3} s t^{3} x^{6}-9 r s t x^{2}+8 r^{2} s^{2} t^{2} x^{5}-r^{2} s^{3} t^{2} x^{6}+4 r s^{2} t x^{3}+2 r^{3} s t x^{3}+7 r s^{3} t x^{4}+2 r s t^{3} x^{5}-2 r s^{4} t x^{5}+r s t^{5} x^{8}\right) W_{1} W_{0}$

and

$\Phi_{1}=x^{n+3}\left(n\left(r+s t x^{2}\right)\left(-t^{2} x^{3}+s x+r t x^{2}+1\right)\left(r^{2} x-s^{2} x^{2}+t^{2} x^{3}+2 s x+2 r t x^{2}-1\right)+2 r^{3} x-3 r-\right.$ $r^{3} t^{2} x^{4}+2 r^{2} t^{3} x^{5}-s^{2} t^{3} x^{6}-5 s t x^{2}+r s^{2} x^{2}+r^{3} s x^{2}+r^{2} t x^{2}+4 s^{2} t x^{3}+3 s^{3} t x^{4}+3 r t^{4} x^{6}+4 s t^{3} x^{5}-2 s^{4}$ $\left.t x^{5}+s t^{5} x^{8}+2 r s x+4 r^{2} s^{2} t x^{4}+6 r s^{2} t^{2} x^{5}+2 r^{3} s t^{2} x^{5}-r s^{3} t^{2} x^{6}+r^{2} s t^{3} x^{6}+4 r^{2} s t x^{3}+4 r s t^{2} x^{4}\right) W_{n+3}^{2}$,

$\Phi_{2}=x^{n+4}(t+r s)\left(n(s+r t x)\left(-t^{2} x^{3}+s x+r t x^{2}+1\right)\left(r^{2} x-s^{2} x^{2}+t^{2} x^{3}+2 s x+2 r t x^{2}-1\right)+3 s^{2} x-\right.$ $4 s+2 s^{3} x^{2}-s^{4} x^{3}+2 r^{2} s^{2} x^{2}+3 r^{2} t^{2} x^{3}+2 r^{4} t^{2} x^{4}+r^{3} t^{3} x^{5}-s^{3} t^{2} x^{5}+3 r^{2} s x+4 r^{3} t x^{2}+4 r t^{3} x^{4}+2 s t^{2} x^{3}+$ $\left.r t^{5} x^{7}+2 s t^{4} x^{6}-5 r t x+8 r^{2} s t^{2} x^{4}+6 r s t x^{2}-r^{2} s^{2} t^{2} x^{5}+7 r s^{2} t x^{3}+4 r^{3} s t x^{3}-2 r s^{3} t x^{4}\right) W_{n+2}^{2}$,

$\Phi_{3}=t^{2} x^{n+4}\left(n\left(r+s t x^{2}\right)\left(-t^{2} x^{3}+s x+r t x^{2}+1\right)\left(r^{2} x-s^{2} x^{2}+t^{2} x^{3}+2 s x+2 r t x^{2}-1\right)+3 r^{3} x-4 r+r^{2} t^{3} x^{5}-\right.$ $2 s^{2} t^{3} x^{6}+s^{3} t^{3} x^{7}-6 s t x^{2}+2 r s^{2} x^{2}+2 r^{3} s x^{2}-r s^{3} x^{3}+2 r^{2} t x^{2}+2 r t^{2} x^{3}+r^{4} t x^{3}+5 s^{2} t x^{3}+4 s^{3} t x^{4}+2 r t^{4} x^{6}+$ $6 s t^{3} x^{5}-3 s^{4} t x^{5}+3 r s x+4 r^{2} s^{2} t x^{4}+11 r s^{2} t^{2} x^{5}+3 r^{3} s t^{2} x^{5}-2 r s^{3} t^{2} x^{6}+2 r^{2} s t^{3} x^{6}+9 r^{2} s t x^{3}+4 r s t^{2} x^{4}-r s t^{4}$ $\left.x^{7}\right) W_{n+1}^{2}$,

$\Phi_{4}=x^{n+2}\left(n\left(t^{2} x^{3}-s x-r t x^{2}-1\right)\left(r^{2} x+s^{2} x^{2}+t^{2} x^{3}+2 r s t x^{3}-1\right)\left(r^{2} x-s^{2} x^{2}+t^{2} x^{3}+2 s x+2 r t x^{2}-1\right)+\right.$ $4 r^{2} x-2 r^{4} x^{2}+4 s^{2} x^{2}-2 s^{3} x^{3}+3 t^{2} x^{3}-2 s^{4} x^{4}+s^{5} x^{5}-t^{6} x^{9}+s x-4 r^{2} s^{2} x^{3}-2 r^{2} s^{3} x^{4}-6 r^{2} t^{2} x^{4}+r^{4} t^{2} x^{5}-$ $8 s^{2} t^{2} x^{5}-4 r^{3} t^{3} x^{6}-4 r^{2} t^{4} x^{7}+2 s^{3} t^{2} x^{6}+s^{4} t^{2} x^{7}-2 s^{2} t^{4} x^{8}-2 r^{2} s x^{2}-r^{4} s x^{3}-2 r^{3} t x^{3}-2 s t^{2} x^{4}+s t^{4} x^{7}-$ $10 r^{2} s t^{2} x^{5}-8 r^{3} s^{2} t x^{5}+2 r s^{2} t^{3} x^{7}-4 r^{4} s t^{2} x^{6}-2 r^{3} s t^{3} x^{7}+6 r s t x^{3}-14 r^{2} s^{2} t^{2} x^{6}+2 r^{2} s^{3} t^{2} x^{7}-8 r s^{2} t x^{4}-8 r^{3}$ $\left.s t x^{4}-10 r s^{3} t x^{5}-16 r s t^{3} x^{6}+4 r s^{4} t x^{6}-2 r s t^{5} x^{9}-2\right) W_{n+3} W_{n+2}$,

$\Phi_{5}=t x^{n+3}\left(n\left(-t^{2} x^{3}+s x+r t x^{2}+1\right)\left(r^{2} x-s^{2} x^{2}-t^{2} x^{3}+1\right)\left(r^{2} x-s^{2} x^{2}+t^{2} x^{3}+2 s x+2 r t x^{2}-1\right)+3 r^{4} x^{2}-\right.$ $2 r^{2} x+6 s^{2} x^{2}-4 s^{3} x^{3}+6 t^{2} x^{3}-3 s^{4} x^{4}+2 s^{5} x^{5}-3 t^{4} x^{6}+2 s x-2 r^{2} s^{2} x^{3}-4 r^{2} s^{3} x^{4}-4 r^{2} t^{2} x^{4}-10 s^{2} t^{2} x^{5}-2 r^{3} t^{3} x^{6}+$ $4 s^{3} t^{2} x^{6}-2 s^{2} t^{4} x^{8}+r t x^{2}-2 r t^{3} x^{5}-4 s t^{2} x^{4}+r^{5} t x^{4}+r t^{5} x^{8}+2 s t^{4} x^{7}-4 r^{2} s t^{2} x^{5}-2 r^{3} s^{2} t x^{5}+2 r s^{2} t^{3} x^{7}-$ $\left.2 r^{2} s^{2} t^{2} x^{6}-2 r s^{2} t x^{4}+4 r^{3} s t x^{4}-8 r s^{3} t x^{5}-12 r s t^{3} x^{6}+r s^{4} t x^{6}-3+4 r^{2} s x^{2}+2 r^{4} s x^{3}+2 r^{3} t x^{3}\right) W_{n+3} W_{n+1}$, 
$\Phi_{6}=x^{n+1}\left(n\left(t^{2} x^{3}-s x-r t x^{2}-1\right)\left(r^{2} x-s^{2} x^{2}+t^{2} x^{3}+2 s x+2 r t x^{2}-1\right)\left(r^{2} x+s^{2} x^{2}-s^{3} x^{3}+t^{2} x^{3}+\right.\right.$ $\left.s x+r t x^{2}+r^{2} s x^{2}+r^{3} t x^{3}-r t^{3} x^{5}-s t^{2} x^{4}+2 r s t x^{3}-r s^{2} t x^{4}-1\right)+2 r^{2} x-r^{4} x^{2}+s^{2} x^{2}-4 s^{3} x^{3}+s^{4} x^{4}+2 s^{5}$ $x^{5}-s^{6} x^{6}+3 t^{4} x^{6}-2 t^{6} x^{9}+2 s x-4 r^{2} s^{2} x^{3}-5 r^{2} t^{2} x^{4}-r^{4} s^{2} x^{4}+2 r^{2} s^{4} x^{5}-4 s^{2} t^{2} x^{5}-2 r^{2} t^{4} x^{7}+8 s^{3} t^{2} x^{6}-r^{6} t^{2} x^{6}+$ $2 r^{4} t^{4} x^{8}-3 s^{2} t^{4} x^{8}-s^{5} t^{2} x^{8}-r^{2} t^{6} x^{10}+2 s^{3} t^{4} x^{9}+2 r t x^{2}-3 s t^{2} x^{4}-2 r^{5} t x^{4}+2 r t^{5} x^{8}+s t^{6} x^{10}-2 r^{4} s x^{3}-4 r t^{3} x^{5}-$ $2 r^{2} s t^{2} x^{5}-4 r^{3} s^{2} t x^{5}+16 r s^{2} t^{3} x^{7}+4 r^{3} s^{3} t x^{6}-5 r^{4} s t^{2} x^{6}+4 r^{3} s t^{3} x^{7}-2 r s^{3} t^{3} x^{8}+8 r^{2} s t^{4} x^{8}+2 r s^{2} t^{5} x^{10}-2 r s t x^{3}+$ $10 r^{2} s^{3} t^{2} x^{7}+2 r^{4} s^{2} t^{2} x^{7}-r^{2} s^{4} t^{2} x^{8}+2 r^{3} s^{2} t^{3} x^{8}-2 r^{2} s^{2} t^{4} x^{9}-6 r s^{2} t x^{4}-6 r^{3} s t x^{4}+8 r s^{4} t x^{6}-2 r^{5} s t x^{5}-2 r s^{5}$ $\left.t x^{7}-6 r s t^{5} x^{9}-1-4 r s t^{3} x^{6}\right) W_{n+2} W_{n+1}$,

$\Phi_{7}=x^{2}\left(2 r-r^{3} x+2 r^{3} t^{2} x^{4}-3 r^{2} t^{3} x^{5}+s^{3} t^{3} x^{7}+4 s t x^{2}-r s^{3} x^{3}+2 r t^{2} x^{3}+r^{4} t x^{3}-3 s^{2} t x^{3}-2 s^{3} t x^{4}-\right.$ $\left.4 r t^{4} x^{6}-2 s t^{3} x^{5}+s^{4} t x^{5}-2 s t^{5} x^{8}-r s x-4 r^{2} s^{2} t x^{4}-r s^{2} t^{2} x^{5}-r^{3} s t^{2} x^{5}+r^{2} s t x^{3}-4 r s t^{2} x^{4}-r s t^{4} x^{7}\right) W_{2}^{2}$,

$\Phi_{8}=x^{3}(t+r s)\left(3 s-2 s^{2} x-s^{3} x^{2}-r^{2} s^{2} x^{2}-2 r^{2} t^{2} x^{3}-r^{4} t^{2} x^{4}-s^{2} t^{2} x^{4}-r^{2} t^{4} x^{6}+2 s^{3} t^{2} x^{5}-2 r^{2} s x-\right.$ $\left.3 r^{3} t x^{2}-2 r t^{3} x^{4}-2 r t^{5} x^{7}-3 s t^{4} x^{6}+4 r t x-3 r^{2} s t^{2} x^{4}+r s^{2} t^{3} x^{6}-4 r s t x^{2}-2 r s^{2} t x^{3}-2 r^{3} s t x^{3}-2 r s t^{3} x^{5}\right) W_{1}^{2}$,

$\Phi_{9}=t^{2} x^{3}\left(3 r-2 r^{3} x+r^{3} t^{2} x^{4}-2 r^{2} t^{3} x^{5}+s^{2} t^{3} x^{6}+5 s t x^{2}-r s^{2} x^{2}-r^{3} s x^{2}-r^{2} t x^{2}-4 s^{2} t x^{3}-3 s^{3} t x^{4}-3 r t^{4} x^{6}-\right.$ $\left.4 s t^{3} x^{5}+2 s^{4} t x^{5}-s t^{5} x^{8}-2 r s x-4 r^{2} s^{2} t x^{4}-6 r s^{2} t^{2} x^{5}-2 r^{3} s t^{2} x^{5}+r s^{3} t^{2} x^{6}-r^{2} s t^{3} x^{6}-4 r^{2} s t x^{3}-4 r s t^{2} x^{4}\right)$ $W_{0}^{2}$

$\Phi_{10}=-x\left(2 r^{2} x-r^{4} x^{2}+2 s^{2} x^{2}-s^{4} x^{4}+3 t^{4} x^{6}-2 t^{6} x^{9}-2 r^{2} s^{2} x^{3}-2 r^{2} s^{3} x^{4}-4 r^{2} t^{2} x^{4}+2 r^{4} t^{2} x^{5}-6 s^{2} t^{2} x^{5}-\right.$ $4 r^{3} t^{3} x^{6}-4 r^{2} t^{4} x^{7}+2 s^{4} t^{2} x^{7}-2 s^{2} t^{4} x^{8}-r t x^{2}-2 r^{2} s x^{2}-2 r^{3} t x^{3}+2 r t^{3} x^{5}+r^{5} t x^{4}-r t^{5} x^{8}-8 r^{2} s t^{2} x^{5}-6 r^{3} s^{2} t x^{5}-$ $2 r s^{2} t^{3} x^{7}-2 r^{4} s t^{2} x^{6}+2 r s^{3} t^{3} x^{8}-2 r^{2} s t^{4} x^{8}-4 r^{2} s^{2} t^{2} x^{6}-4 r s^{2} t x^{4}-2 r^{3} s t x^{4}-4 r s^{3} t x^{5}-8 r s t^{3} x^{6}+r s^{4} t x^{6}-$ $\left.4 r s t^{5} x^{9}-1\right) W_{2} W_{1}$,

$\Phi_{11}=t x^{2}\left(2 r^{2} x-2 r^{4} x^{2}-4 s^{2} x^{2}+2 s^{3} x^{3}-3 t^{2} x^{3}+2 s^{4} x^{4}-s^{5} x^{5}+t^{6} x^{9}-s x+2 r^{2} s^{2} x^{3}+2 r^{2} s^{3} x^{4}+\right.$ $6 r^{2} t^{2} x^{4}+r^{4} t^{2} x^{5}+8 s^{2} t^{2} x^{5}-2 r^{2} t^{4} x^{7}-2 s^{3} t^{2} x^{6}-s^{4} t^{2} x^{7}+2 s^{2} t^{4} x^{8}-2 r^{2} s x^{2}-r^{4} s x^{3}+2 s t^{2} x^{4}-s t^{4} x^{7}+$ $\left.2 r^{2} s t^{2} x^{5}+4 r s t x^{3}+2 r^{2} s^{2} t^{2} x^{6}+4 r s^{3} t x^{5}+8 r s t^{3} x^{6}+2\right) W_{2} W_{0}$,

$\Phi_{12}=t x^{3}\left(3 t-6 t^{3} x^{3}+3 t^{5} x^{6}+6 r s-2 r^{3} s^{2} x^{2}-2 r^{3} t^{2} x^{3}+4 r^{2} t^{3} x^{4}-r^{5} t^{2} x^{4}+2 s^{2} t^{3} x^{5}+2 r^{3} t^{4} x^{6}-\right.$ $2 s^{3} t^{3} x^{6}-4 r s^{2} x-4 r^{3} s x+2 r^{2} t x-2 r s^{3} x^{2}-r t^{2} x^{2}-3 r^{4} t x^{2}+4 s^{2} t x^{2}-4 s^{3} t x^{3}+2 r t^{4} x^{5}+4 s t^{3} x^{4}-3 s^{4} t x^{4}+$ $2 s^{5} t x^{5}-r t^{6} x^{8}-2 s t^{5} x^{7}-2 s t x-6 r^{2} s^{2} t x^{3}-6 r s^{2} t^{2} x^{4}-4 r^{2} s^{3} t x^{4}-2 r^{3} s t^{2} x^{4}-4 r s^{3} t^{2} x^{5}+r s^{4} t^{2} x^{6}-2 r s^{2} t^{4} x^{7}-$ $\left.2 r^{3} s^{2} t^{2} x^{5}-6 r^{2} s t x^{2}-2 r^{4} s t x^{3}+6 r s t^{4} x^{6}\right) W_{1} W_{0}$

and

$\Psi_{1}=\left(n\left(-t^{2} x^{3}+s x+r t x^{2}+1\right)\left(s-s^{2} x+r^{2}+r t x\right)\left(r^{2} x-s^{2} x^{2}+t^{2} x^{3}+2 s x+2 r t x^{2}-1\right)+2 r^{4} x-3 s+\right.$ $6 s^{2} x-2 s^{3} x^{2}-2 s^{4} x^{3}+s^{5} x^{4}-3 r^{2}-r^{2} s^{2} x^{2}-2 r^{2} s^{3} x^{3}+2 r^{2} t^{2} x^{3}-s^{2} t^{2} x^{4}+2 r^{3} t^{3} x^{5}+4 r^{2} t^{4} x^{6}-2 s^{3} t^{2} x^{5}+$ $s^{4} t^{2} x^{6}-2 s^{2} t^{4} x^{7}+4 r^{2} s x+r^{4} s x^{2}+4 r^{3} t x^{2}+2 r t^{3} x^{4}+2 r t^{5} x^{7}+3 s t^{4} x^{6}-4 r t x+4 r^{2} s t^{2} x^{4}-2 r s^{2} t^{3} x^{6}+4 r s$ $\left.t x^{2}-2 r^{2} s^{2} t^{2} x^{5}+2 r^{3} s t x^{3}-4 r s^{3} t x^{4}+2 r s t^{3} x^{5}\right) x^{n+3} W_{n+3}^{2}$,

$\Psi_{2}=\left(n\left(t^{2} x^{3}-s x-r t x^{2}-1\right)\left(r^{2} x-s^{2} x^{2}+t^{2} x^{3}+2 s x+2 r t x^{2}-1\right)\left(-s+s^{2} x-r^{2} t^{2} x^{3}+r^{2} s x-r t^{3} x^{4}+r s^{2} t x^{3}\right)\right.$ $+4 s^{2} x-2 s-2 s^{3} x^{2}-4 r^{2} s^{2} x^{2}-2 r^{2} s^{3} x^{3}-5 r^{2} t^{2} x^{3}-r^{4} s^{2} x^{3}+4 r^{4} t^{2} x^{4}+2 s^{2} t^{2} x^{4}+8 r^{3} t^{3} x^{5}+8 r^{2} t^{4} x^{6}-$ $4 s^{3} t^{2} x^{5}+2 r^{5} t^{3} x^{6}+2 s^{4} t^{2} x^{6}+4 r^{4} t^{4} x^{7}-3 s^{2} t^{4} x^{7}+2 r^{3} t^{5} x^{8}-2 r^{4} s x^{2}-6 r t^{3} x^{4}-2 s t^{2} x^{3}+6 r t^{5} x^{7}+4 s t^{4} x^{6}+$ $2 r^{2} s t^{2} x^{4}-4 r^{3} s^{2} t x^{4}-2 r s^{2} t^{3} x^{6}-4 r^{3} s^{3} t x^{5}+4 r^{4} s t^{2} x^{5}+10 r^{3} s t^{3} x^{6}-2 r s^{3} t^{3} x^{7}+8 r^{2} s t^{4} x^{7}-8 r^{2} s^{3} t^{2} x^{6}-2 r^{4} s^{2}$ $t^{2} x^{6}+r^{2} s^{4} t^{2} x^{7}-2 r^{3} s^{2} t^{3} x^{7}-2 r^{2} s^{2} t^{4} x^{8}-2 r^{3} s t x^{3}-2 r s^{3} t x^{4}+8 r s t^{3} x^{5}-4 r s^{4} t x^{5}+2 r s^{5} t x^{6}-2 r s t^{5} x^{8}+$ $\left.4 r^{2} s x\right) x^{n+2} W_{n+2}^{2}$,

$\Psi_{3}=\left(n\left(-t^{2} x^{3}+s x+r t x^{2}+1\right)\left(r^{2} x-s^{2} x^{2}+t^{2} x^{3}+2 s x+2 r t x^{2}-1\right)\left(s-s^{2} x+r^{2}+r t x\right)+3 r^{4} x-\right.$ $4 s+8 s^{2} x-2 s^{3} x^{2}-4 s^{4} x^{3}+2 s^{5} x^{4}-4 r^{2}-4 r^{2} s^{3} x^{3}+5 r^{2} t^{2} x^{3}+2 r^{4} t^{2} x^{4}-4 s^{2} t^{2} x^{4}+2 r^{3} t^{3} x^{5}+2 r^{2} t^{4} x^{6}-s^{2}$ 
$t^{4} x^{7}+6 r^{2} s x+2 r^{4} s x^{2}+6 r^{3} t x^{2}+4 r t^{3} x^{4}+2 s t^{2} x^{3}+r^{5} t x^{3}+r t^{5} x^{7}+2 s t^{4} x^{6}-5 r t x+8 r^{2} s t^{2} x^{4}-2 r^{3} s^{2} t x^{4}+$ $\left.6 r s t x^{2}-3 r^{2} s^{2} t^{2} x^{5}+4 r s^{2} t x^{3}+8 r^{3} s t x^{3}-10 r s^{3} t x^{4}+r s^{4} t x^{5}\right) t^{2} x^{n+4} W_{n+1}^{2}$,

$\Psi_{4}=\left(n(r+t x)\left(t^{2} x^{3}-s x-r t x^{2}-1\right)\left(r^{2} x-s^{2} x^{2}+t^{2} x^{3}-1\right)\left(r^{2} x-s^{2} x^{2}+t^{2} x^{3}+2 s x+2 r t x^{2}-1\right)+\right.$ $4 r^{3} x-2 r-2 r^{5} x^{2}+6 t^{3} x^{4}-3 t^{5} x^{7}-3 t x+2 r^{3} s^{2} x^{3}+2 r^{3} s^{3} x^{4}-8 r^{3} t^{2} x^{4}-8 r^{2} t^{3} x^{5}-4 r^{4} t^{3} x^{6}-2 s^{2} t^{3} x^{6}-$ $8 r^{3} t^{4} x^{7}-4 r^{2} t^{5} x^{8}+2 s^{3} t^{3} x^{7}+2 s t x^{2}-4 r s^{2} x^{2}-2 r^{3} s x^{2}+4 r s^{3} x^{3}+6 r^{2} t x^{2}+4 r t^{2} x^{3}+2 r s^{4} x^{4}-r^{5} s x^{3}-5 r^{4} t$ $x^{3}-4 s^{2} t x^{3}-r s^{5} x^{5}+4 s^{3} t x^{4}-2 r t^{4} x^{6}-4 s t^{3} x^{5}+3 s^{4} t x^{5}-2 s^{5} t x^{6}+2 s t^{5} x^{8}+r s x+6 r^{2} s^{2} t x^{4}+8 r^{2} s^{3} t x^{5}-$ $8 r^{3} s t^{2} x^{5}+10 r s^{3} t^{2} x^{6}-12 r^{2} s t^{3} x^{6}-2 r s^{4} t^{2} x^{7}+4 r s^{2} t^{4} x^{8}+4 r^{3} s^{2} t^{2} x^{6}+4 r^{2} s^{2} t^{3} x^{7}-6 r^{2} s t x^{3}-2 r s t^{2} x^{4}-$ $\left.2 r^{4} s t x^{4}-11 r s t^{4} x^{7}\right) x^{n+2} W_{n+3} W_{n+2}$,

$\Psi_{5}=\left(n\left(t^{2} x^{3}-s x-r t x^{2}-1\right)\left(r^{2} x+s^{2} x^{2}-s^{3} x^{3}+t^{2} x^{3}+s x+r^{2} s x^{2}-s t^{2} x^{4}+2 r s t x^{3}-1\right)\left(r^{2} x-s^{2} x^{2}+\right.\right.$ $\left.t^{2} x^{3}+2 s x+2 r t x^{2}-1\right)+2 r^{2} x-r^{4} x^{2}+s^{2} x^{2}-4 s^{3} x^{3}+s^{4} x^{4}+2 s^{5} x^{5}-s^{6} x^{6}+3 t^{4} x^{6}-2 t^{6} x^{9}+2 s x-4 r^{2} s^{2} x^{3}-$ $4 r^{2} t^{2} x^{4}-r^{4} s^{2} x^{4}+2 r^{2} s^{4} x^{5}+2 r^{4} t^{2} x^{5}-4 s^{2} t^{2} x^{5}-4 r^{3} t^{3} x^{6}-4 r^{2} t^{4} x^{7}+8 s^{3} t^{2} x^{6}-3 s^{2} t^{4} x^{8}-s^{5} t^{2} x^{8}+2 s^{3} t^{4} x^{9}-r t$ $x^{2}-2 r^{4} s x^{3}-2 r^{3} t x^{3}+2 r t^{3} x^{5}-3 s t^{2} x^{4}+r^{5} t x^{4}-r t^{5} x^{8}+s t^{6} x^{10}-2 r^{2} s t^{2} x^{5}-6 r^{3} s^{2} t x^{5}+6 r s^{2} t^{3} x^{7}-r^{4} s t^{2}$ $x^{6}+2 r s^{3} t^{3} x^{8}-4 r^{2} s t^{4} x^{8}-2 r^{2} s^{2} t^{2} x^{6}+2 r^{2} s^{3} t^{2} x^{7}-2 r^{3} s t x^{4}-4 r s^{3} t x^{5}-8 r s t^{3} x^{6}+5 r s^{4} t x^{6}-4 r s t^{5} x^{9}-$ 1) $x^{n+1} W_{n+3} W_{n+1}$,

$\Psi_{6}=\left(n(s x-1)\left(-t^{2} x^{3}+s x+r t x^{2}+1\right)\left(r^{2} x-s^{2} x^{2}+t^{2} x^{3}+2 s x+2 r t x^{2}-1\right)\left(r^{2} x-s^{2} x^{2}+t^{2} x^{3}-1\right)+4 r^{2} x-\right.$ $2 r^{4} x^{2}-6 s^{2} x^{2}+8 s^{3} x^{3}+3 t^{2} x^{3}-2 s^{4} x^{4}-4 s^{5} x^{5}+2 s^{6} x^{6}-t^{6} x^{9}+4 s x+4 r^{2} s^{2} x^{3}-6 r^{2} t^{2} x^{4}+2 r^{4} s^{2} x^{4}-4 r^{2} s^{4} x^{5}+r^{4} t^{2}$ $x^{5}-4 r^{3} t^{3} x^{6}-4 r^{2} t^{4} x^{7}+4 s^{3} t^{2} x^{6}-3 s^{4} t^{2} x^{7}-8 r^{2} s x^{2}+2 r^{4} s x^{3}-2 r^{3} t x^{3}-8 s t^{2} x^{4}+4 s t^{4} x^{7}+4 r^{2} s t^{2} x^{5}+4 r^{3} s^{2} t x^{5}+$ $14 r s^{2} t^{3} x^{7}-2 r^{3} s^{3} t x^{6}+4 r^{3} s t^{3} x^{7}-2 r s^{3} t^{3} x^{8}+4 r^{2} s t^{4} x^{8}-5 r s t x^{3}+6 r^{2} s^{2} t^{2} x^{6}-2 r^{2} s^{3} t^{2} x^{7}+4 r s^{2} t x^{4}+2$ $\left.r^{3} s t x^{4}-6 r s t^{3} x^{6}-8 r s^{4} t x^{6}+r^{5} s t x^{5}+r s^{5} t x^{7}-r s t^{5} x^{9}-2\right) t x^{n+2} W_{n+2} W_{n+1}$,

$\Psi_{7}=\left(2 s-r^{4} x-4 s^{2} x+2 s^{3} x^{2}+2 r^{2}+2 r^{2} s^{2} x^{2}+r^{2} t^{2} x^{3}+2 r^{4} t^{2} x^{4}-2 s^{2} t^{2} x^{4}-2 r^{3} t^{3} x^{5}-6 r^{2} t^{4} x^{6}+\right.$ $4 s^{3} t^{2} x^{5}-2 s^{4} t^{2} x^{6}+3 s^{2} t^{4} x^{7}-2 r^{2} s x-2 r^{3} t x^{2}+2 s t^{2} x^{3}+r^{5} t x^{3}-3 r t^{5} x^{7}-4 s t^{4} x^{6}+3 r t x-2 r^{3} s^{2} t x^{4}+4 r s^{2} t^{3} x^{6}-$ $\left.2 r s t x^{2}+r^{2} s^{2} t^{2} x^{5}+4 r s^{2} t x^{3}+4 r^{3} s t x^{3}-2 r s^{3} t x^{4}-4 r s t^{3} x^{5}+r s^{4} t x^{5}\right) x^{2} W_{2}^{2}$

$\Psi_{8}=x\left(s-2 s^{2} x+2 s^{3} x^{2}-2 s^{4} x^{3}+s^{5} x^{4}+3 r^{2} s^{2} x^{2}+4 r^{2} t^{2} x^{3}+r^{2} s^{4} x^{4}-3 r^{4} t^{2} x^{4}-5 s^{2} t^{2} x^{4}-6 r^{3} t^{3} x^{5}-\right.$ $5 r^{2} t^{4} x^{6}+6 s^{3} t^{2} x^{5}-r^{5} t^{3} x^{6}-3 s^{4} t^{2} x^{6}-2 r^{4} t^{4} x^{7}+4 s^{2} t^{4} x^{7}-2 r^{3} t^{5} x^{8}-2 r^{2} t^{6} x^{9}-2 r^{2} s x+r^{4} s x^{2}+5 r t^{3} x^{4}+$ $4 s t^{2} x^{3}-4 r t^{5} x^{7}-5 s t^{4} x^{6}-r t^{7} x^{10}-2 r^{2} s t^{2} x^{4}-2 r^{3} s^{2} t x^{4}+2 r s^{2} t^{3} x^{6}+4 r^{3} s^{3} t x^{5}-4 r^{4} s t^{2} x^{5}-4 r^{3} s t^{3} x^{6}+$ $2 r s^{3} t^{3} x^{7}-4 r^{2} s t^{4} x^{7}-r s^{4} t^{3} x^{8}+2 r s^{2} t^{5} x^{9}+r s t x^{2}+2 r^{2} s^{3} t^{2} x^{6}+r^{4} s^{2} t^{2} x^{6}+3 r^{2} s^{2} t^{4} x^{8}+4 r s^{2} t x^{3}+2 r^{3} s t x^{3}-$ $\left.4 r s^{3} t x^{4}-8 r s t^{3} x^{5}+4 r s^{4} t x^{5}-r^{5} s t x^{4}-r s^{5} t x^{6}+r s t^{5} x^{8}\right) W_{1}^{2}$,

$\Psi_{9}=t^{2} x^{3}\left(3 s-2 r^{4} x-6 s^{2} x+2 s^{3} x^{2}+2 s^{4} x^{3}-s^{5} x^{4}+3 r^{2}+r^{2} s^{2} x^{2}+2 r^{2} s^{3} x^{3}-2 r^{2} t^{2} x^{3}+s^{2} t^{2} x^{4}-\right.$ $2 r^{3} t^{3} x^{5}-4 r^{2} t^{4} x^{6}+2 s^{3} t^{2} x^{5}-s^{4} t^{2} x^{6}+2 s^{2} t^{4} x^{7}-4 r^{2} s x-r^{4} s x^{2}-4 r^{3} t x^{2}-2 r t^{3} x^{4}-2 r t^{5} x^{7}-3 s t^{4} x^{6}+4 r t x-$ $\left.4 r^{2} s t^{2} x^{4}+2 r s^{2} t^{3} x^{6}-4 r s t x^{2}+2 r^{2} s^{2} t^{2} x^{5}-2 r^{3} s t x^{3}+4 r s^{3} t x^{4}-2 r s t^{3} x^{5}\right) W_{0}^{2}$,

$\Psi_{10}=x\left(-r^{6} t x^{4}-2 r^{5} t^{2} x^{5}+r^{5} x^{2}+2 r^{4} s^{2} t x^{5}-3 r^{4} s t x^{4}+3 r^{4} t^{3} x^{6}+4 r^{4} t x^{3}-2 r^{3} s^{2} t^{2} x^{6}-2 r^{3} s^{2} x^{3}+\right.$ $4 r^{3} s t^{2} x^{5}+2 r^{3} s x^{2}+8 r^{3} t^{4} x^{7}+6 r^{3} t^{2} x^{4}-2 r^{3} x-r^{2} s^{4} t x^{6}-2 r^{2} s^{3} t x^{5}-4 r^{2} s^{2} t^{3} x^{7}-6 r^{2} s^{2} t x^{4}+8 r^{2} s t^{3} x^{6}+10 r^{2}$ $s t x^{3}+5 r^{2} t^{5} x^{8}+4 r^{2} t^{3} x^{5}-3 r^{2} t x^{2}+2 r s^{4} t^{2} x^{7}-r s^{4} x^{4}-6 r s^{3} t^{2} x^{6}-4 r s^{3} x^{3}-6 r s^{2} t^{4} x^{8}+2 r s^{2} t^{2} x^{5}+4 r s^{2} x^{2}+$ $8 r s t^{4} x^{7}+4 r s t^{2} x^{4}+2 r t^{6} x^{9}-3 r t^{4} x^{6}+r+s^{5} t x^{6}+s^{4} t^{3} x^{8}-2 s^{4} t x^{5}-2 s^{3} t^{3} x^{7}-4 s^{3} t x^{4}-2 s^{2} t^{5} x^{9}+4 s^{2} t^{3} x^{6}+$ $\left.4 s^{2} t x^{3}-s t^{5} x^{8}+2 s t^{3} x^{5}-s t x^{2}+t^{7} x^{10}-3 t^{3} x^{4}+2 t x\right) W_{2} W_{1}$,

$\Psi_{11}=t x^{2}\left(2 r+2 r^{3} x-2 r^{5} x^{2}-6 t^{3} x^{4}+3 t^{5} x^{7}+3 t x+2 r^{3} s^{3} x^{4}+4 r^{3} t^{2} x^{4}+4 r^{2} t^{3} x^{5}+2 s^{2} t^{3} x^{6}-2 s^{3} t^{3} x^{7}-2 s t x^{2}-\right.$ $8 r s^{2} x^{2}-6 r^{3} s x^{2}+2 r^{2} t x^{2}-4 r t^{2} x^{3}+2 r s^{4} x^{4}-r^{5} s x^{3}-3 r^{4} t x^{3}+4 s^{2} t x^{3}-r s^{5} x^{5}-4 s^{3} t x^{4}+2 r t^{4} x^{6}+4 s t^{3} x^{5}-3 s^{4} t x^{5}+$ $\left.2 s^{5} t x^{6}-2 s t^{5} x^{8}+5 r s x-6 r^{2} s^{2} t x^{4}+2 r s^{2} t^{2} x^{5}-6 r s^{3} t^{2} x^{6}+8 r^{2} s t^{3} x^{6}-2 r^{2} s t x^{3}+2 r s t^{2} x^{4}-2 r^{4} s t x^{4}+5 r s t^{4} x^{7}\right)$ $W_{2} W_{0}$, 


$$
\begin{aligned}
& \Psi_{12}=t x\left(-r^{5} t x^{4}-r^{4} s^{2} x^{4}+r^{4} s t^{2} x^{6}-2 r^{4} s x^{3}-2 r^{4} t^{2} x^{5}+r^{4} x^{2}+2 r^{3} s^{2} t x^{5}-4 r^{3} s t^{3} x^{7}-6 r^{3} s t x^{4}+\right. \\
& 4 r^{3} t^{3} x^{6}+2 r^{3} t x^{3}+2 r^{2} s^{4} x^{5}+2 r^{2} s^{3} t^{2} x^{7}+2 r^{2} s^{3} x^{4}-6 r^{2} s^{2} t^{2} x^{6}-4 r^{2} s^{2} x^{3}-4 r^{2} s t^{4} x^{8}-2 r^{2} s t^{2} x^{5}+6 r^{2} s x^{2}+4 r^{2} \\
& t^{4} x^{7}+4 r^{2} t^{2} x^{4}-2 r^{2} x+3 r s^{4} t x^{6}+2 r s^{3} t^{3} x^{8}+4 r s^{3} t x^{5}-10 r s^{2} t^{3} x^{7}-8 r s^{2} t x^{4}+4 r s t^{3} x^{6}+8 r s t x^{3}+r t^{5} x^{8}- \\
& 2 r t^{3} x^{5}+r t x^{2}-s^{6} x^{6}-s^{5} t^{2} x^{8}+2 s^{5} x^{5}+4 s^{4} t^{2} x^{7}+3 s^{4} x^{4}+2 s^{3} t^{4} x^{9}-6 s^{3} t^{2} x^{6}-8 s^{3} x^{3}-3 s^{2} t^{4} x^{8}+4 s^{2} t^{2} x^{5}+ \\
& \left.5 s^{2} x^{2}-s t^{6} x^{10}+3 s t^{2} x^{4}-2 s x+2 t^{6} x^{9}-3 t^{4} x^{6}+1\right) W_{1} W_{0} .
\end{aligned}
$$

Proof. First, we obtain $\sum_{k=0}^{n} k x^{k} W_{k}^{2}$. Using the recurrence relation

$$
W_{n+3}=r W_{n+2}+s W_{n+1}+t W_{n}
$$

or

$$
t W_{n}=W_{n+3}-r W_{n+2}-s W_{n+1}
$$

i.e.

$t^{2} W_{n}^{2}=\left(W_{n+3}-r W_{n+2}-s W_{n+1}\right)^{2}=W_{n+3}^{2}+r^{2} W_{n+2}^{2}+s^{2} W_{n+1}^{2}-2 r W_{n+3} W_{n+2}-2 s W_{n+3} W_{n+1}+2 r s W_{n+2} W_{n+1}$

we obtain

$$
\begin{aligned}
t^{2} \times n \times x^{n} W_{n}^{2}= & n \times x^{n} W_{n+3}^{2}+r^{2} \times n \times x^{n} W_{n+2}^{2}+s^{2} \times n \times x^{n} W_{n+1}^{2} \\
& -2 r \times n \times x^{n} W_{n+3} W_{n+2}-2 s \times n \times x^{n} W_{n+3} W_{n+1}+2 r s \times n \times x^{n} W_{n+2} W_{n+1} \\
t^{2} \times(n-1) \times x^{n-1} W_{n-1}^{2}= & (n-1) \times x^{n-1} W_{n+2}^{2}+r^{2} \times(n-1) \times x^{n-1} W_{n+1}^{2}+s^{2} \times(n-1) \times x^{n-1} W_{n}^{2} \\
& -2 r \times(n-1) \times x^{n-1} W_{n+2} W_{n+1}-2 s \times(n-1) \times x^{n-1} W_{n+2} W_{n} \\
& +2 r s \times(n-1) \times x^{n-1} W_{n+1} W_{n} \\
t^{2} \times(n-2) \times x^{n-2} W_{n-2}^{2}=\quad & (n-2) \times x^{n-2} W_{n+1}^{2}+r^{2} \times(n-2) x^{n-2} W_{n}^{2}+s^{2} \times(n-2) \times x^{n-2} W_{n-1}^{2} \\
& -2 r \times(n-2) \times x^{n-2} W_{n+1} W_{n}-2 s \times(n-2) \times x^{n-2} W_{n+1} W_{n-1} \\
& +2 r s \times(n-2) x^{n-2} W_{n} W_{n-1} \\
& \vdots \\
t^{2} \times 1 \times x^{1} W_{1}^{2}= & 1 \times x^{1} W_{4}^{2}+r^{2} \times 1 \times x^{1} W_{3}^{2}+s^{2} \times 1 \times x^{1} W_{2}^{2} \\
& -2 r \times 1 \times x^{1} W_{4} W_{3}-2 s \times 1 \times x^{1} W_{4} W_{2}+2 r s \times 1 \times x^{1} W_{3} W_{2} \\
t^{2} \times 0 \times x^{0} W_{0}^{2}= & 0 \times x^{0} W_{3}^{2}+r^{2} \times 0 \times x^{0} W_{2}^{2}+s^{2} \times 0 \times x^{0} W_{1}^{2} \\
& -2 r \times 0 \times x^{0} W_{3} W_{2}-2 s \times 0 \times x^{0} W_{3} W_{1}+2 r s \times 0 \times x^{0} W_{2} W_{1}
\end{aligned}
$$


If we add the equations side by side, we get

$$
\begin{aligned}
t^{2} \sum_{k=0}^{n} k x^{k} W_{k}^{2}= & \left(n x^{n} W_{n+3}^{2}+(n-1) x^{n-1} W_{n+2}^{2}+(n-2) x^{n-2} W_{n+1}^{2}\right. \\
& \left.+1 \times x^{-1} W_{2}^{2}+2 \times x^{-2} W_{1}^{2}+3 \times x^{-3} W_{0}^{2}+\sum_{k=0}^{n}(k-3) x^{k-3} W_{k}^{2}\right) \\
& +r^{2}\left(n x^{n} W_{n+2}^{2}+(n-1) x^{n-1} W_{n+1}^{2}+1 \times x^{-1} W_{1}^{2}+2 \times x^{-2} W_{0}^{2}+\sum_{k=0}^{n}(k-2) x^{k-2} W_{k}^{2}\right) \\
& +s^{2}\left(n x^{n} W_{n+1}^{2}+1 \times x^{-1} W_{0}^{2}+\sum_{k=0}^{n}(k-1) x^{k-1} W_{k}^{2}\right)-2 r\left(n x^{n} W_{n+3} W_{n+2}\right. \\
& \left.+(n-1) x^{n-1} W_{n+2} W_{n+1}+1 \times x^{-1} W_{2} W_{1}+2 \times x^{-2} W_{1} W_{0}+\sum_{k=0}^{n}(k-2) x^{k-2} W_{k+1} W_{k}\right) \\
& -2 s\left(n x^{n} W_{n+3} W_{n+1}+1 \times x^{-1} W_{2} W_{0}+\sum_{k=0}^{n}(k-1) x^{k-1} W_{k+2} W_{k}\right) \\
& +2 r s\left(n x^{n} W_{n+2} W_{n+1}+1 \times x^{-1} W_{1} W_{0}+\sum_{k=0}^{n}(k-1) x^{k-1} W_{k+1} W_{k}\right)
\end{aligned}
$$

and so

$$
\begin{aligned}
t^{2} \sum_{k=0}^{n} k x^{k} W_{k}^{2}= & x^{-3}\left(r^{2} x+s^{2} x^{2}+1\right) \sum_{k=0}^{n} k x^{k} W_{k}^{2}-x^{-3}\left(2 r^{2} x+s^{2} x^{2}+3\right) \sum_{k=0}^{n} x^{k} W_{k}^{2} \\
& -2 s x^{-1} \sum_{k=0}^{n} k x^{k} W_{k} W_{k+2}+2 s x^{-1} \sum_{k=0}^{n} x^{k} W_{k} W_{k+2}+2 r(s x-1) x^{-2} \sum_{k=0}^{n} k x^{k} W_{k+1} W_{k} \\
& +2 r(2-s x) x^{-2} \sum_{k=0}^{n} x^{k} W_{k+1} W_{k}+n x^{n} W_{n+3}^{2}+\left(n+n r^{2} x-1\right) x^{n-1} W_{n+2}^{2} \\
& +\left(n-r^{2} x+n r^{2} x+n s^{2} x^{2}-2\right) x^{n-2} W_{n+1}^{2}-2 n r x^{n} W_{n+2} W_{n+3} \\
& -2 n s x^{n} W_{n+1} W_{n+3}+2 r(-n+n s x+1) x^{n-1} W_{n+1} W_{n+2} \\
& +x^{-1} W_{2}^{2}+\left(r^{2} x+2\right) x^{-2} W_{1}^{2}+\left(2 r^{2} x+s^{2} x^{2}+3\right) x^{-3} W_{0}^{2} \\
& -2 r x^{-1} W_{1} W_{2}-2 s x^{-1} W_{0} W_{2}+2 r(s x-2) x^{-2} W_{1} W_{0}
\end{aligned}
$$

Next we obtain $\sum_{k=0}^{n} k x^{k} W_{k+1} W_{k}$. Multiplying the both side of the recurrence relation

$$
t W_{n}=W_{n+3}-r W_{n+2}-s W_{n+1}
$$

by $W_{n+1}$ we get

$$
t W_{n+1} W_{n}=W_{n+3} W_{n+1}-r W_{n+2} W_{n+1}-s W_{n+1}^{2} .
$$


Then using last recurrence relation, we obtain

$$
\begin{aligned}
t \times n \times x^{n} W_{n+1} W_{n}= & n x^{n} W_{n+3} W_{n+1}-r \times n \times x^{n} W_{n+2} W_{n+1}-s \times n \times x^{n} W_{n+1}^{2} \\
t \times(n-1) \times x^{n-1} W_{n} W_{n-1}= & (n-1) \times x^{n-1} W_{n+2} W_{n}-r \times(n-1) \times x^{n-1} W_{n+1} W_{n} \\
& -s \times(n-1) \times x^{n-1} W_{n}^{2} \\
t \times(n-2) \times x^{n-2} W_{n-1} W_{n-2}= & (n-2) \times x^{n-2} W_{n+1} W_{n-1}-r \times(n-2) \times x^{n-2} W_{n} W_{n-1} \\
& -s \times(n-2) \times x^{n-2} W_{n-1}^{2} \\
& \vdots \\
t \times 2 \times x^{2} W_{3} W_{2}= & 2 \times x^{2} W_{5} W_{3}-r \times 2 \times W_{4} W_{3}-s \times 2 \times x^{2} W_{3}^{2} \\
t \times 1 \times x W_{2} W_{1}= & 1 \times x W_{4} W_{2}-r \times 1 \times x W_{3} W_{2}-s \times 1 \times x W_{2}^{2} \\
t \times 0 \times x^{0} W_{1} W_{0}= & 0 \times x^{0} W_{3} W_{1}-r \times 0 \times x^{0} W_{2} W_{1}-s \times 0 \times x^{0} W_{1}^{2}
\end{aligned}
$$

If we add the equations side by side, we get

$$
\begin{aligned}
t \sum_{k=0}^{n} k x^{k} W_{k+1} W_{k}= & \left(n x^{n} W_{n+3} W_{n+1}+1 \times x^{-1} W_{2} W_{0}+\sum_{k=0}^{n}(k-1) x^{k-1} W_{k+2} W_{k}\right) \\
& -r\left(n x^{n} W_{n+2} W_{n+1}+1 \times x^{-1} W_{1} W_{0}+\sum_{k=0}^{n}(k-1) x^{k-1} W_{k+1} W_{k}\right) \\
& -s\left(n x^{n} W_{n+1}^{2}+1 \times x^{-1} W_{0}^{2}+\sum_{k=0}^{n}(k-1) x^{k-1} W_{k}^{2}\right)
\end{aligned}
$$

and so

$$
\begin{aligned}
t \sum_{k=0}^{n} k x^{k} W_{k+1} W_{k}= & -s x^{-1} \sum_{k=0}^{n} k x^{k} W_{k}^{2}+s x^{-1} \sum_{k=0}^{n} x^{k} W_{k}^{2}+x^{-1} \sum_{k=0}^{n} k x^{k} W_{k} W_{k+2} \\
& -x^{-1} \sum_{k=0}^{n} x^{k} W_{k} W_{k+2}-r x^{-1} \sum_{k=0}^{n} k x^{k} W_{k} W_{k+1}+r x^{-1} \sum_{k=0}^{n} x^{k} W_{k} W_{k+1} \\
& -n s x^{n} W_{n+1}^{2}+n x^{n} W_{n+3} W_{n+1}-n r x^{n} W_{n+2} W_{n+1}-\frac{s}{x} W_{0}^{2}+\frac{1}{x} W_{2} W_{0}-\frac{r}{x} W_{1} W_{0}
\end{aligned}
$$

Next we obtain $\sum_{k=0}^{n} k x^{k} W_{k+2} W_{k}$. Multiplying the both side of the recurrence relation

$$
t W_{n}=W_{n+3}-r W_{n+2}-s W_{n+1}
$$

by $W_{n+2}$ we get

$$
t W_{n+2} W_{n}=W_{n+3} W_{n+2}-r W_{n+2}^{2}-s W_{n+2} W_{n+1} .
$$


Then using last recurrence relation, we obtain

$$
\begin{aligned}
t \times n \times x^{n} W_{n+2} W_{n}= & n x^{n} W_{n+3} W_{n+2}-r \times n \times x^{n} W_{n+2}^{2}-s \times n \times x^{n} W_{n+2} W_{n+1} \\
t \times(n-1) \times x^{n-1} W_{n+1} W_{n-1}= & (n-1) \times x^{n-1} W_{n+2} W_{n+1}-r \times(n-1) \times x^{n-1} W_{n+1}^{2} \\
& -s \times(n-1) \times x^{n-1} W_{n+1} W_{n} \\
t \times(n-2) x^{n-2} W_{n} W_{n-2}= & (n-2) \times x^{n-2} W_{n+1} W_{n}-r \times(n-2) \times x^{n-2} W_{n}^{2}-s \times(n-2) \times x^{n-2} W_{n} W_{n-1} \\
& \vdots \\
t \times 2 \times x^{2} W_{4} W_{2}= & 2 \times x^{2} W_{5} W_{4}-r \times 2 \times x^{2} W_{4}^{2}-s \times 2 \times x^{2} W_{4} W_{3} \\
t \times 1 \times x^{1} W_{3} W_{1}= & 1 \times x^{1} W_{4} W_{3}-r \times 1 \times x^{1} W_{3}^{2}-s \times 1 \times x^{1} W_{3} W_{2} \\
t \times 0 \times x^{0} W_{2} W_{0}= & 0 \times x^{0} W_{3} W_{2}-r \times 0 \times x^{0} W_{2}^{2}-s \times 0 \times x^{0} W_{2} W_{1}
\end{aligned}
$$

If we add the equations side by side, we get

$$
\begin{aligned}
t \sum_{k=0}^{n} k x^{k} W_{k+2} W_{k}= & \left(n x^{n} W_{n+3} W_{n+2}+(n-1) x^{n-1} W_{n+2} W_{n+1}+1 \times x^{-1} W_{2} W_{1}\right. \\
& \left.+2 \times x^{-2} W_{1} W_{0}+\sum_{k=0}^{n}(k-2) x^{k-2} W_{k+1} W_{k}\right)-r\left(n x^{n} W_{n+2}^{2}+(n-1) x^{n-1} W_{n+1}^{2}\right. \\
& \left.+1 \times x^{-1} W_{1}^{2}+2 \times x^{-2} W_{0}^{2}+\sum_{k=0}^{n}(k-2) x^{k-2} W_{k}^{2}\right)-s\left(n x^{n} W_{n+2} W_{n+1}\right. \\
& \left.+1 \times x^{-1} W_{1} W_{0}+\sum_{k=0}^{n}(k-1) x^{k-1} W_{k+1} W_{k}\right)
\end{aligned}
$$

and so

$$
\begin{aligned}
t \sum_{k=0}^{n} k x^{k} W_{k+2} W_{k}= & -r x^{-2} \sum_{k=0}^{n} k x^{k} W_{k}^{2}+2 r x^{-2} \sum_{k=0}^{n} x^{k} W_{k}^{2}-(s x-1) x^{-2} \sum_{k=0}^{n} k x^{k} W_{k+1} W_{k} \\
& +(s x-2) x^{-2} \sum_{k=0}^{n} x^{k} W_{k+1} W_{k}-n r x^{n} W_{n+2}^{2}-r(n-1) x^{n-1} W_{n+1}^{2} \\
& +n x^{n} W_{n+3} W_{n+2}+(n-n s x-1) x^{n-1} W_{n+2} W_{n+1}-\frac{r}{x} W_{1}^{2} \\
& -2 \frac{r}{x^{2}} W_{0}^{2}+\frac{1}{x} W_{2} W_{1}-(s x-2) x^{-2} W_{1} W_{0}
\end{aligned}
$$

Using Theorem 1.1 and solving the system (2.1)-(2.2)-(2.3), the results in (a), (b) and (c) follow.

\section{Specific Cases}

In this section, we present the closed form solutions (identities) of the sums $\sum_{k=0}^{n} k x^{k} W_{k}^{2}, \sum_{k=0}^{n} k x^{k} W_{k+2} W_{k}$ and $\sum_{k=0}^{n} k x^{k} W_{k+1} W_{k}$ for the specific case of sequence $\left\{W_{n}\right\}$.

3.1. The Case $x=1$. The case $x=1$ of Theorem 2.1 is given in [30]. In this subsection, we only consider the case $x=1, r=0, s=2, t=1$ and $x=1, r=1, s=1, t=2$ (these two special cases were not given in [30] because we can not use Theorem 2.1 directly). Observe that setting $x=1, r=0, s=2, t=1$ and $x=1, r=1, s=1, t=2$ (i.e. for the generalized Pell-Padovan case and for the generalized third order Jacobsthal case) in Theorem 2.1 (a), (b) and (c) makes the right hand side of the sum formulas to be an indeterminate form. Application of L'Hospital rule (using twice) however provides the evaluation of the sum formulas. If $x=1, r=0, s=2, t=1$ then we have the 
following theorem (in fact taking $x=1, r=0, s=2, t=1$ in Theorem 2.1 and then using L'Hospital rule twice for $x=1$ we obtain the following theorem).

Theorem 3.1. If $r=0, s=2, t=1$ then for $n \geq 0$ we have the following formulas:

(a): $\sum_{k=0}^{n} k W_{k}^{2}=\frac{1}{4}\left(\left(2 n^{2}+18 n+69\right) W_{n+3}^{2}+\left(2 n^{2}+14 n+53\right) W_{n+2}^{2}+\left(2 n^{2}+18 n+85\right) W_{n+1}^{2}-4\left(n^{2}+8 n+\right.\right.$ $31) W_{n+3} W_{n+2}-4\left(n^{2}+10 n+40\right) W_{n+3} W_{n+1}+4\left(n^{2}+10 n+38\right) W_{n+2} W_{n+1}-53 W_{2}^{2}-41 W_{1}^{2}-69 W_{0}^{2}-$ $\left.116 W_{1} W_{0}+124 W_{2} W_{0}+96 W_{2} W_{1}\right)$.

(b): $\sum_{k=0}^{n} k W_{k+1} W_{k}=\frac{1}{4}\left(-2\left(n^{2}+8 n+31\right) W_{n+3}^{2}-2\left(n^{2}+6 n+24\right) W_{n+2}^{2}-2\left(n^{2}+10 n+40\right) W_{n+1}^{2}+\left(4 n^{2}+\right.\right.$ $30 n+111) W_{n+3} W_{n+2}+\left(4 n^{2}+38 n+145\right) W_{n+3} W_{n+1}-\left(4 n^{2}+38 n+137\right) W_{n+2} W_{n+1}+48 W_{2}^{2}+38 W_{1}^{2}+62$ $\left.W_{0}^{2}-85 W_{2} W_{1}-111 W_{2} W_{0}+103 W_{1} W_{0}\right)$.

(c): $\sum_{k=0}^{n} k W_{k+2} W_{k}=\frac{1}{4}\left(2\left(n^{2}+8 n+29\right) W_{n+3}^{2}+2\left(n^{2}+6 n+22\right) W_{n+2}^{2}+2\left(n^{2}+10 n+38\right) W_{n+1}^{2}-\left(4 n^{2}+26 n+\right.\right.$ 103) $W_{n+3} W_{n+2}-\left(4 n^{2}+38 n+137\right) W_{n+3} W_{n+1}+\left(4 n^{2}+34 n+125\right) W_{n+2} W_{n+1}-44 W_{2}^{2}-34 W_{1}^{2}-58 W_{0}^{2}+81$ $\left.W_{2} W_{1}+103 W_{2} W_{0}-95 W_{1} W_{0}\right)$.

From Theorem 3.1, we have the following corollary which gives sum formulas of Pell-Padovan numbers (take $W_{n}=R_{n}$ with $\left.Q_{0}=1, R_{1}=1, R_{2}=1\right)$.

Corollary 3.2. For $n \geq 0$, Pell-Padovan numbers have the following properties:

(a): $\sum_{k=0}^{n} k R_{k}^{2}=\frac{1}{4}\left(\left(2 n^{2}+18 n+69\right) R_{n+3}^{2}+\left(2 n^{2}+14 n+53\right) R_{n+2}^{2}+\left(2 n^{2}+18 n+85\right) R_{n+1}^{2}-4\left(n^{2}+8 n+\right.\right.$ 31) $\left.R_{n+3} R_{n+2}-4\left(n^{2}+10 n+40\right) R_{n+3} R_{n+1}+4\left(n^{2}+10 n+38\right) R_{n+2} R_{n+1}-59\right)$.

(b): $\sum_{k=0}^{n} k R_{k+1} R_{k}=\frac{1}{4}\left(-2\left(n^{2}+8 n+31\right) R_{n+3}^{2}-2\left(n^{2}+6 n+24\right) R_{n+2}^{2}-2\left(n^{2}+10 n+40\right) R_{n+1}^{2}+\left(4 n^{2}+\right.\right.$ $\left.30 n+111) R_{n+3} R_{n+2}+\left(4 n^{2}+38 n+145\right) R_{n+3} R_{n+1}-\left(4 n^{2}+38 n+137\right) R_{n+2} R_{n+1}+55\right)$.

(c): $\sum_{k=0}^{n} k R_{k+2} R_{k}=\frac{1}{4}\left(2\left(n^{2}+8 n+29\right) R_{n+3}^{2}+2\left(n^{2}+6 n+22\right) R_{n+2}^{2}+2\left(n^{2}+10 n+38\right) R_{n+1}^{2}-\left(4 n^{2}+26 n+\right.\right.$ 103) $\left.R_{n+3} R_{n+2}-\left(4 n^{2}+38 n+137\right) R_{n+3} R_{n+1}+\left(4 n^{2}+34 n+125\right) R_{n+2} R_{n+1}-47\right)$.

Taking $R_{n}=C_{n}$ with $C_{0}=3, C_{1}=0, C_{2}=2$ in Theorem 3.1, we have the following corollary which presents sum formulas of Pell-Perrin numbers.

Corollary 3.3. For $n \geq 0$, Pell-Perrin numbers have the following properties:

(a): $\sum_{k=0}^{n} k C_{k}^{2}=\frac{1}{4}\left(\left(2 n^{2}+18 n+69\right) C_{n+3}^{2}+\left(2 n^{2}+14 n+53\right) C_{n+2}^{2}+\left(2 n^{2}+18 n+85\right) C_{n+1}^{2}-4\left(n^{2}+8 n+\right.\right.$ 31) $\left.C_{n+3} C_{n+2}-4\left(n^{2}+10 n+40\right) C_{n+3} C_{n+1}+4\left(n^{2}+10 n+38\right) C_{n+2} C_{n+1}-89\right)$.

(b): $\sum_{k=0}^{n} k C_{k+1} C_{k}=\frac{1}{4}\left(-2\left(n^{2}+8 n+31\right) C_{n+3}^{2}-2\left(n^{2}+6 n+24\right) C_{n+2}^{2}-2\left(n^{2}+10 n+40\right) C_{n+1}^{2}+\left(4 n^{2}+30 n+\right.\right.$ 111) $\left.C_{n+3} C_{n+2}+\left(4 n^{2}+38 n+145\right) C_{n+3} C_{n+1}-\left(4 n^{2}+38 n+137\right) C_{n+2} C_{n+1}+84\right)$.

(c): $\sum_{k=0}^{n} k C_{k+2} C_{k}=\frac{1}{4}\left(2\left(n^{2}+8 n+29\right) C_{n+3}^{2}+2\left(n^{2}+6 n+22\right) C_{n+2}^{2}+2\left(n^{2}+10 n+38\right) C_{n+1}^{2}-\left(4 n^{2}+26 n+\right.\right.$ 103) $\left.C_{n+3} C_{n+2}-\left(4 n^{2}+38 n+137\right) C_{n+3} C_{n+1}+\left(4 n^{2}+34 n+125\right) C_{n+2} C_{n+1}-80\right)$.

If $x=1, r=1, s=1, t=2$ then we have the following theorem (in fact taking $r=1, s=1, t=2$ in Theorem 2.1 and then using L'Hospital rule twice for $x=1$ we obtain the following theorem).

TheOREM 3.4. If $r=1, s=1, t=2$ then for $n \geq 0$ we have the following formulas:

(a): $\sum_{k=0}^{n} k W_{k}^{2}=\frac{1}{1323}\left(\left(63 n^{2}+198 n-4076\right) W_{n+3}^{2}+9\left(21 n^{2}+31 n-1381\right) W_{n+2}^{2}+\left(252 n^{2}-27 n-16583\right) W_{n+1}^{2}-\right.$ $9\left(21 n^{2}+45 n-1366\right) W_{n+3} W_{n+2}-2\left(63 n^{2}+135 n-4070\right) W_{n+3} W_{n+1}+12(21 n+19) W_{n+2} W_{n+1}+4211 W_{2}^{2}+$ $\left.12519 W_{1}^{2}+16304 W_{0}^{2}-12510 W_{1} W_{2}-8284 W_{2} W_{0}+24 W_{1} W_{0}\right)$. 
(b): $\sum_{k=0}^{n} k W_{k+1} W_{k}=\frac{1}{2646}\left(-\left(63 n^{2}+9 n-4142\right) W_{n+3}^{2}-3\left(63 n^{2}+51 n-4174\right) W_{n+2}^{2}-4\left(63 n^{2}+135 n-\right.\right.$ $4070) W_{n+1}^{2}+3\left(63 n^{2}+93 n-4192\right) W_{n+3} W_{n+2}+2\left(63 n^{2}+387 n-3968\right) W_{n+3} W_{n+1}-6(189 n+73) W_{n+2} W_{n+1}-$ $\left.4088 W_{2}^{2}-12486 W_{1}^{2}-16568 W_{0}^{2}+12666 W_{2} W_{1}+8584 W_{0} W_{2}-696 W_{0} W_{1}\right)$.

(c): $\sum_{k=0}^{n} k W_{k+2} W_{k}=\frac{1}{2646}\left(-\left(63 n^{2}-117 n-4130\right) W_{n+3}^{2}-9\left(21 n^{2}+73 n-1336\right) W_{n+2}^{2}-4\left(63 n^{2}+9 n-\right.\right.$ $4184) W_{n+1}^{2}+27\left(7 n^{2}+29 n-452\right) W_{n+3} W_{n+2}+2\left(63 n^{2}-180 n-4187\right) W_{n+3} W_{n+1}-12(21 n+40) W_{n+2} W_{n+1}-$ $\left.3950 W_{2}^{2}-12492 W_{1}^{2}-16520 W_{0}^{2}+12798 W_{2} W_{1}+7888 W_{2} W_{0}+228 W_{1} W_{0}\right)$.

From Theorem 3.4, we have the following corollary which gives sum formulas of third order Jacobsthal numbers (take $W_{n}=J_{n}$ with $J_{0}=0, J_{1}=1, J_{2}=1$ ).

Corollary 3.5. For $n \geq 0$, third order Jacobsthal numbers have the following properties:

(a): $\sum_{k=0}^{n} k J_{k}^{2}=\frac{1}{1323}\left(\left(63 n^{2}+198 n-4076\right) J_{n+3}^{2}+9\left(21 n^{2}+31 n-1381\right) J_{n+2}^{2}+\left(252 n^{2}-27 n-16583\right) J_{n+1}^{2}-\right.$ $\left.9\left(21 n^{2}+45 n-1366\right) J_{n+3} J_{n+2}-2\left(63 n^{2}+135 n-4070\right) J_{n+3} J_{n+1}+12(21 n+19) J_{n+2} J_{n+1}+4220\right)$.

(b): $\sum_{k=0}^{n} k J_{k+1} J_{k}=\frac{1}{2646}\left(-\left(63 n^{2}+9 n-4142\right) J_{n+3}^{2}-3\left(63 n^{2}+51 n-4174\right) J_{n+2}^{2}-4\left(63 n^{2}+135 n-4070\right) J_{n+1}^{2}+\right.$ $\left.3\left(63 n^{2}+93 n-4192\right) J_{n+3} J_{n+2}+2\left(63 n^{2}+387 n-3968\right) J_{n+3} J_{n+1}-6(189 n+73) J_{n+2} J_{n+1}-3908\right)$.

(c): $\sum_{k=0}^{n} k J_{k+2} J_{k}=\frac{1}{2646}\left(-\left(63 n^{2}-117 n-4130\right) J_{n+3}^{2}-9\left(21 n^{2}+73 n-1336\right) J_{n+2}^{2}-4\left(63 n^{2}+9 n-4184\right) J_{n+1}^{2}+\right.$ $\left.27\left(7 n^{2}+29 n-452\right) J_{n+3} J_{n+2}+2\left(63 n^{2}-180 n-4187\right) J_{n+3} J_{n+1}-12(21 n+40) J_{n+2} J_{n+1}-3644\right)$.

Taking $W_{n}=j_{n}$ with $j_{0}=2, j_{1}=1, j_{2}=5$ in Theorem 3.4, we have the following corollary which presents sum formulas of third order Jacobsthal-Lucas numbers.

Corollary 3.6. For $n \geq 0$, third order Jacobsthal-Lucas numbers have the following properties:

(a): $\sum_{k=0}^{n} k j_{k}^{2}=\frac{1}{1323}\left(\left(63 n^{2}+198 n-4076\right) j_{n+3}^{2}+9\left(21 n^{2}+31 n-1381\right) j_{n+2}^{2}+\left(252 n^{2}-27 n-16583\right) j_{n+1}^{2}-\right.$ $\left.9\left(21 n^{2}+45 n-1366\right) j_{n+3} j_{n+2}-2\left(63 n^{2}+135 n-4070\right) j_{n+3} j_{n+1}+12(21 n+19) j_{n+2} j_{n+1}+37668\right)$.

(b): $\sum_{k=0}^{n} k j_{k+1} j_{k}=\frac{1}{2646}\left(-\left(63 n^{2}+9 n-4142\right) j_{n+3}^{2}-3\left(63 n^{2}+51 n-4174\right) j_{n+2}^{2}-4\left(63 n^{2}+135 n-4070\right) j_{n+1}^{2}+\right.$ $\left.3\left(63 n^{2}+93 n-4192\right) j_{n+3} j_{n+2}+2\left(63 n^{2}+387 n-3968\right) j_{n+3} j_{n+1}-6(189 n+73) j_{n+2} j_{n+1}-33180\right)$.

(c): $\sum_{k=0}^{n} k j_{k+2} j_{k}=\frac{1}{2646}\left(-\left(63 n^{2}-117 n-4130\right) j_{n+3}^{2}-9\left(21 n^{2}+73 n-1336\right) j_{n+2}^{2}-4\left(63 n^{2}+9 n-4184\right) j_{n+1}^{2}+\right.$ $\left.27\left(7 n^{2}+29 n-452\right) j_{n+3} j_{n+2}+2\left(63 n^{2}-180 n-4187\right) j_{n+3} j_{n+1}-12(21 n+40) j_{n+2} j_{n+1}-33996\right)$.

3.2. The Case $x=-1$. We now consider the case $x=-1$ in Theorem 2.1 .

Taking $x=-1, r=s=t=1$ in Theorem 2.1, we obtain the following Proposition.

Proposition 3.7. If $x=-1, r=s=t=1$ then for $n \geq 0$ we have the following formulas:

(a): $\sum_{k=0}^{n} k(-1)^{k} W_{k}^{2}=\frac{1}{4}\left((-1)^{n}\left((n+1) W_{n+3}^{2}-(2 n+1) W_{n+2}^{2}+(3 n+2) W_{n+1}^{2}-2(n+2) W_{n+1} W_{n+3}+\right.\right.$ $\left.\left.2 W_{n+2} W_{n+1}\right)+W_{1}^{2}-W_{0}^{2}-2 W_{2} W_{0}+2 W_{1} W_{0}\right)$.

(b): $\sum_{k=0}^{n} k(-1)^{k} W_{k+1} W_{k}=\frac{1}{4}\left((-1)^{n}\left((n+1) W_{n+3}^{2}+W_{n+2}^{2}-(n+2) W_{n+1}^{2}-(2 n+2) W_{n+3} W_{n+2}+2 n W_{n+2} W_{n+1}\right)+\right.$ $\left.W_{1}^{2}-W_{0}^{2}-2 W_{1} W_{0}\right)$.

(c): $\sum_{k=0}^{n} k(-1)^{k} W_{k+2} W_{k}=\frac{1}{4}\left((-1)^{n}\left(n W_{n+3}^{2}-(2 n+1) W_{n+2}^{2}-(n+1) W_{n+1}^{2}+2 W_{n+3} W_{n+2}+2 n W_{n+3} W_{n+1}-\right.\right.$ $\left.\left.4(n+1) W_{n+2} W_{n+1}\right)-W_{2}^{2}+W_{1}^{2}+2 W_{2} W_{1}-2 W_{2} W_{0}\right)$.

From Proposition 3.7, we have the following Corollary which gives sum formulas of Tribonacci numbers (take $W_{n}=T_{n}$ with $\left.T_{0}=0, T_{1}=1, T_{2}=1\right)$. 
Corollary 3.8. For $n \geq 0$, Tribonacci numbers have the following properties:

(a): $\sum_{k=0}^{n} k(-1)^{k} T_{k}^{2}=\frac{1}{4}\left((-1)^{n}\left((n+1) T_{n+3}^{2}-(2 n+1) T_{n+2}^{2}+(3 n+2) T_{n+1}^{2}-2(n+2) T_{n+1} T_{n+3}+2 T_{n+2} T_{n+1}\right)+\right.$ 1).

(b): $\sum_{k=0}^{n} k(-1)^{k} T_{k+1} T_{k}=\frac{1}{4}\left((-1)^{n}\left((n+1) T_{n+3}^{2}+T_{n+2}^{2}-(n+2) T_{n+1}^{2}-(2 n+2) T_{n+3} T_{n+2}+2 n T_{n+2} T_{n+1}\right)+\right.$ $1)$.

(c): $\sum_{k=0}^{n} k(-1)^{k} T_{k+2} T_{k}=\frac{1}{4}\left((-1)^{n}\left(n T_{n+3}^{2}-(2 n+1) T_{n+2}^{2}-(n+1) T_{n+1}^{2}+2 T_{n+3} T_{n+2}+2 n T_{n+3} T_{n+1}-\right.\right.$ $\left.\left.4(n+1) T_{n+2} T_{n+1}\right)+2\right)$.

Taking $T_{n}=K_{n}$ with $K_{0}=3, K_{1}=1, K_{2}=3$ in Proposition 3.7, we have the following Corollary which presents sum formulas of Tribonacci-Lucas numbers.

Corollary 3.9. For $n \geq 0$, Tribonacci-Lucas numbers have the following properties:

(a): $\sum_{k=0}^{n} k(-1)^{k} K_{k}^{2}=\frac{1}{4}\left((-1)^{n}\left((n+1) K_{n+3}^{2}-(2 n+1) K_{n+2}^{2}+(3 n+2) K_{n+1}^{2}-2(n+2) K_{n+1} K_{n+3}+\right.\right.$ $\left.\left.2 K_{n+2} K_{n+1}\right)-20\right)$.

(b): $\sum_{k=0}^{n} k(-1)^{k} K_{k+1} K_{k}=\frac{1}{4}\left((-1)^{n}\left((n+1) K_{n+3}^{2}+K_{n+2}^{2}-(n+2) K_{n+1}^{2}-(2 n+2) K_{n+3} K_{n+2}+2 n K_{n+2} K_{n+1}\right)-\right.$ 14)

(c): $\sum_{k=0}^{n} k(-1)^{k} K_{k+2} K_{k}=\frac{1}{4}\left((-1)^{n}\left(n K_{n+3}^{2}-(2 n+1) K_{n+2}^{2}-(n+1) K_{n+1}^{2}+2 K_{n+3} K_{n+2}+2 n K_{n+3} K_{n+1}-\right.\right.$ $\left.\left.4(n+1) K_{n+2} K_{n+1}\right)-20\right)$.

Taking $x=-1, r=2, s=1, t=1$ in Theorem 2.1, we obtain the following Proposition.

Proposition 3.10. If $r=2, s=1, t=1$ then for $n \geq 0$ we have the following formulas:

(a): $\sum_{k=0}^{n} k(-1)^{k} W_{k}^{2}=\frac{1}{75}\left((-1)^{n}\left((5 n+2) W_{n+3}^{2}-(45 n+53) W_{n+2}^{2}+(70 n+68) W_{n+1}^{2}+2(5 n+12) W_{n+3} W_{n+2}-2\right.\right.$ $\left.\left.(15 n+31) W_{n+3} W_{n+1}+2(10 n+39) W_{n+2} W_{n+1}\right)-3 W_{2}^{2}-8 W_{1}^{2}-2 W_{0}^{2}+14 W_{2} W_{1}-32 W_{0} W_{2}+58 W_{1} W_{0}\right)$.

(b): $\sum_{k=0}^{n} k(-1)^{k} W_{k+1} W_{k}=\frac{1}{75}\left((-1)^{n}\left((15 n+16) W_{n+3}^{2}+3(5 n+17) W_{n+2}^{2}-(15 n+31) W_{n+1}^{2}-(45 n+58) W_{n+3} W_{n+2}-\right.\right.$ $\left.\left.(15 n+21) W_{n+3} W_{n+1}+(60 n+49) W_{n+2} W_{n+1}\right)+W_{2}^{2}+36 W_{1}^{2}-16 W_{0}^{2}-13 W_{1} W_{2}-6 W_{2} W_{0}-11 W_{1} W_{0}\right)$. (c): $\sum_{k=0}^{n} k(-1)^{k} W_{k+2} W_{k}=\frac{1}{75}\left((-1)^{n}(20 n+3) W_{n+3}^{2}-(-1)^{n}(30 n+17) W_{n+2}^{2}-(-1)^{n}(20 n+23) W_{n+1}^{2}-(-1)^{n}\right.$ $(35 n-11) W_{n+3} W_{n+2}+(-1)^{n}(30 n+7) W_{n+3} W_{n+1}-(-1)^{n}(70 n+83) W_{n+2} W_{n+1}-17 W_{2}^{2}+13 W_{1}^{2}-3 W_{0}^{2}+$ $\left.46 W_{1} W_{2}-23 W_{2} W_{0}-13 W_{1} W_{0}\right)$.

From Proposition 3.10, we have the following Corollary which gives sum formulas of Third-order Pell numbers (take $W_{n}=P_{n}$ with $\left.P_{0}=0, P_{1}=1, P_{2}=1\right)$.

Corollary 3.11. For $n \geq 0$, third-order Pell numbers have the following properties:

(a): $\sum_{k=0}^{n} k(-1)^{k} P_{k}^{2}=\frac{1}{75}\left((-1)^{n}\left((5 n+2) P_{n+3}^{2}-(45 n+53) P_{n+2}^{2}+(70 n+68) P_{n+1}^{2}+2(5 n+12) P_{n+3} P_{n+2}-2\right.\right.$ $\left.\left.(15 n+31) P_{n+3} P_{n+1}+2(10 n+39) P_{n+2} P_{n+1}\right)+8\right)$.

(b): $\sum_{k=0}^{n} k(-1)^{k} P_{k+1} P_{k}=\frac{1}{75}\left((-1)^{n}\left((15 n+16) P_{n+3}^{2}+3(5 n+17) P_{n+2}^{2}-(15 n+31) P_{n+1}^{2}-(45 n+58) P_{n+3} P_{n+2}-\right.\right.$ $\left.\left.(15 n+21) P_{n+3} P_{n+1}+(60 n+49) P_{n+2} P_{n+1}\right)+14\right)$.

(c): $\sum_{k=0}^{n} k(-1)^{k} P_{k+2} P_{k}=\frac{1}{75}\left((-1)^{n}(20 n+3) P_{n+3}^{2}-(-1)^{n}(30 n+17) P_{n+2}^{2}-(-1)^{n}(20 n+23) P_{n+1}^{2}-(-1)^{n}\right.$ $\left.(35 n-11) P_{n+3} P_{n+2}+(-1)^{n}(30 n+7) P_{n+3} P_{n+1}-(-1)^{n}(70 n+83) P_{n+2} P_{n+1}+37\right)$

Taking $W_{n}=Q_{n}$ with $Q_{0}=3, Q_{1}=2, Q_{2}=6$ in Proposition 3.10, we have the following Corollary which presents sum formulas of third-order Pell-Lucas numbers. 
Corollary 3.12. For $n \geq 0$, third-order Pell-Lucas numbers have the following properties:

(a): $\sum_{k=0}^{n} k(-1)^{k} Q_{k}^{2}=\frac{1}{75}\left((-1)^{n}\left((5 n+2) Q_{n+3}^{2}-(45 n+53) Q_{n+2}^{2}+(70 n+68) Q_{n+1}^{2}+2(5 n+12) Q_{n+3} Q_{n+2}-2\right.\right.$ $\left.\left.(15 n+31) Q_{n+3} Q_{n+1}+2(10 n+39) Q_{n+2} Q_{n+1}\right)-218\right)$.

(b): $\sum_{k=0}^{n} k(-1)^{k} Q_{k+1} Q_{k}=\frac{1}{75}\left((-1)^{n}\left((15 n+16) Q_{n+3}^{2}+3(5 n+17) Q_{n+2}^{2}-(15 n+31) Q_{n+1}^{2}-(45 n+58) Q_{n+3} Q_{n+2}-\right.\right.$ $\left.\left.(15 n+21) Q_{n+3} Q_{n+1}+(60 n+49) Q_{n+2} Q_{n+1}\right)-294\right)$.

(c): $\sum_{k=0}^{n} k(-1)^{k} Q_{k+2} Q_{k}=\frac{1}{75}\left((-1)^{n}(20 n+3) Q_{n+3}^{2}-(-1)^{n}(30 n+17) Q_{n+2}^{2}-(-1)^{n}(20 n+23) Q_{n+1}^{2}-(-1)^{n}\right.$ $\left.(35 n-11) Q_{n+3} Q_{n+2}+(-1)^{n}(30 n+7) Q_{n+3} Q_{n+1}-(-1)^{n}(70 n+83) Q_{n+2} Q_{n+1}-527\right)$.

From Proposition 3.10, we have the following Corollary which gives sum formulas of third-order modified Pell numbers (take $W_{n}=E_{n}$ with $\left.E_{0}=0, E_{1}=1, E_{2}=1\right)$.

Corollary 3.13. For $n \geq 0$, third-order modified Pell numbers have the following properties:

(a): $\sum_{k=0}^{n} k(-1)^{k} E_{k}^{2}=\frac{1}{75}\left((-1)^{n}\left((5 n+2) E_{n+3}^{2}-(45 n+53) E_{n+2}^{2}+(70 n+68) E_{n+1}^{2}+2(5 n+12) E_{n+3} E_{n+2}-2\right.\right.$ $\left.\left.(15 n+31) E_{n+3} E_{n+1}+2(10 n+39) E_{n+2} E_{n+1}\right)+3\right)$.

(b): $\sum_{k=0}^{n} k(-1)^{k} E_{k+1} E_{k}=\frac{1}{75}\left((-1)^{n}\left((15 n+16) E_{n+3}^{2}+3(5 n+17) E_{n+2}^{2}-(15 n+31) E_{n+1}^{2}-(45 n+58) E_{n+3} E_{n+2}-\right.\right.$ $\left.\left.(15 n+21) E_{n+3} E_{n+1}+(60 n+49) E_{n+2} E_{n+1}\right)+24\right)$.

(c): $\sum_{k=0}^{n} k(-1)^{k} E_{k+2} E_{k}=\frac{1}{75}\left((-1)^{n}(20 n+3) E_{n+3}^{2}-(-1)^{n}(30 n+17) E_{n+2}^{2}-(-1)^{n}(20 n+23) E_{n+1}^{2}-(-1)^{n}\right.$ $\left.(35 n-11) E_{n+3} E_{n+2}+(-1)^{n}(30 n+7) E_{n+3} E_{n+1}-(-1)^{n}(70 n+83) E_{n+2} E_{n+1}+42\right)$.

Taking $x=-1, r=0, s=1, t=1$ in Theorem 2.1, we obtain the following Proposition.

Proposition 3.14. If $r=0, s=1, t=1$ then for $n \geq 0$ we have the following formulas:

(a): $\sum_{k=0}^{n} k(-1)^{k} W_{k}^{2}=\frac{1}{25}\left((-1)^{n}\left((15 n+14) W_{n+3}^{2}-(15 n-1) W_{n+2}^{2}+(10 n-4) W_{n+1}^{2}+2(5 n+8) W_{n+3} W_{n+2}-2\right.\right.$ $\left.\left.(10 n+11) W_{n+2} W_{n+1}-2(5 n+13) W_{n+3} W_{n+1}\right)-W_{2}^{2}+16 W_{1}^{2}-14 W_{0}^{2}+6 W_{1} W_{2}-16 W_{2} W_{0}-2 W_{1} W_{0}\right)$.

(b): $\sum_{k=0}^{n} k(-1)^{k} W_{k+1} W_{k}=\frac{1}{25}\left((-1)^{n}\left((5 n+8) W_{n+3}^{2}-(5 n+3) W_{n+2}^{2}-(5 n+13) W_{n+1}^{2}-(5 n-2) W_{n+3} W_{n+2}+\right.\right.$ $\left.\left.(10 n-9) W_{n+2} W_{n+1}+(5 n+3) W_{n+3} W_{n+1}\right)+3 W_{2}^{2}+2 W_{1}^{2}-8 W_{0}^{2}+7 W_{2} W_{1}-2 W_{2} W_{0}-19 W_{1} W_{0}\right)$.

(c): $\sum_{k=0}^{n} k(-1)^{k} W_{k+2} W_{k}=\frac{1}{25}\left((-1)^{n}\left((10 n+1) W_{n+3}^{2}-(10 n-9) W_{n+2}^{2}-(10 n+11) W_{n+1}^{2}+(15 n+19) W_{n+3} W_{n+2}+\right.\right.$ $\left.\left.(10 n-9) W_{n+3} W_{n+1}-(30 n+23) W_{n+2} W_{n+1}\right)-9 W_{2}^{2}+19 W_{1}^{2}-W_{0}^{2}+4 W_{2} W_{1}-19 W_{2} W_{0}+7 W_{1} W_{0}\right)$.

From Proposition 3.14, we have the following Corollary which gives sum formulas of Padovan numbers (take $W_{n}=P_{n}$ with $\left.P_{0}=1, P_{1}=1, P_{2}=1\right)$.

Corollary 3.15. For $n \geq 0$, Padovan numbers have the following properties:

(a): $\sum_{k=0}^{n} k(-1)^{k} P_{k}^{2}=\frac{1}{25}\left((-1)^{n}\left((15 n+14) P_{n+3}^{2}-(15 n-1) P_{n+2}^{2}+(10 n-4) P_{n+1}^{2}+2(5 n+8) P_{n+3} P_{n+2}-2\right.\right.$ $\left.\left.(10 n+11) P_{n+2} P_{n+1}-2(5 n+13) P_{n+3} P_{n+1}\right)-11\right)$.

(b): $\sum_{k=0}^{n} k(-1)^{k} P_{k+1} P_{k}=\frac{1}{25}\left((-1)^{n}\left((5 n+8) P_{n+3}^{2}-(5 n+3) P_{n+2}^{2}-(5 n+13) P_{n+1}^{2}-(5 n-2) P_{n+3} P_{n+2}+\right.\right.$ $\left.\left.(10 n-9) P_{n+2} P_{n+1}+(5 n+3) P_{n+3} P_{n+1}\right)-17\right)$.

(c): $\sum_{k=0}^{n} k(-1)^{k} P_{k+2} P_{k}=\frac{1}{25}\left((-1)^{n}\left((10 n+1) P_{n+3}^{2}-(10 n-9) P_{n+2}^{2}-(10 n+11) P_{n+1}^{2}+(15 n+19) P_{n+3} P_{n+2}+\right.\right.$ $\left.\left.(10 n-9) P_{n+3} P_{n+1}-(30 n+23) P_{n+2} P_{n+1}\right)+1\right)$.

Taking $W_{n}=E_{n}$ with $E_{0}=3, E_{1}=0, E_{2}=2$ in Proposition 3.14, we have the following Corollary which presents sum formulas of Perrin numbers. 
Corollary 3.16. For $n \geq 0$, Perrin numbers have the following properties:

(a): $\sum_{k=0}^{n} k(-1)^{k} E_{k}^{2}=\frac{1}{25}\left((-1)^{n}\left((15 n+14) E_{n+3}^{2}-(15 n-1) E_{n+2}^{2}+(10 n-4) E_{n+1}^{2}+2(5 n+8) E_{n+3} E_{n+2}-2\right.\right.$ $\left.\left.(10 n+11) E_{n+2} E_{n+1}-2(5 n+13) E_{n+3} E_{n+1}\right)-226\right)$.

(b): $\sum_{k=0}^{n} k(-1)^{k} E_{k+1} E_{k}=\frac{1}{25}\left((-1)^{n}\left((5 n+8) E_{n+3}^{2}-(5 n+3) E_{n+2}^{2}-(5 n+13) E_{n+1}^{2}-(5 n-2) E_{n+3} E_{n+2}+\right.\right.$ $\left.\left.(10 n-9) E_{n+2} E_{n+1}+(5 n+3) E_{n+3} E_{n+1}\right)-72\right)$.

(c): $\sum_{k=0}^{n} k(-1)^{k} E_{k+2} E_{k}=\frac{1}{25}\left((-1)^{n}\left((10 n+1) E_{n+3}^{2}-(10 n-9) E_{n+2}^{2}-(10 n+11) E_{n+1}^{2}+(15 n+19) E_{n+3} E_{n+2}+\right.\right.$ $\left.\left.(10 n-9) E_{n+3} E_{n+1}-(30 n+23) E_{n+2} E_{n+1}\right)-159\right)$.

From Proposition 3.14, we have the following Corollary which gives sum formulas of Padovan-Perrin numbers (take $W_{n}=S_{n}$ with $S_{0}=0, S_{1}=0, S_{2}=1$ ).

Corollary 3.17. For $n \geq 0$, Padovan-Perrin numbers have the following properties:

(a): $\sum_{k=0}^{n} k(-1)^{k} S_{k}^{2}=\frac{1}{25}\left((-1)^{n}\left((15 n+14) S_{n+3}^{2}-(15 n-1) S_{n+2}^{2}+(10 n-4) S_{n+1}^{2}+2(5 n+8) S_{n+3} S_{n+2}-2\right.\right.$ $\left.\left.(10 n+11) S_{n+2} S_{n+1}-2(5 n+13) S_{n+3} S_{n+1}\right)-1\right)$.

(b): $\sum_{k=0}^{n} k(-1)^{k} S_{k+1} S_{k}=\frac{1}{25}\left((-1)^{n}\left((5 n+8) S_{n+3}^{2}-(5 n+3) S_{n+2}^{2}-(5 n+13) S_{n+1}^{2}-(5 n-2) S_{n+3} S_{n+2}+\right.\right.$ $\left.\left.(10 n-9) S_{n+2} S_{n+1}+(5 n+3) S_{n+3} S_{n+1}\right)+3\right)$

(c): $\sum_{k=0}^{n} k(-1)^{k} S_{k+2} S_{k}=\frac{1}{25}\left((-1)^{n}\left((10 n+1) S_{n+3}^{2}-(10 n-9) S_{n+2}^{2}-(10 n+11) S_{n+1}^{2}+(15 n+19) S_{n+3} S_{n+2}+\right.\right.$ $\left.\left.(10 n-9) S_{n+3} S_{n+1}-(30 n+23) S_{n+2} S_{n+1}\right)-9\right)$.

Observe that setting $x=-1, r=0, s=2, t=1$ (i.e. for the generalized Pell-Padovan case) in Theorem 2.1 (a), (b) and (c) makes the right hand side of the sum formulas to be an indeterminate form. Application of L'Hospital rule (using twice) however provides the evaluation of the sum formulas. If $x=-1, r=0, s=2, t=1$ then we have the following theorem (in fact taking $x=-1, r=0, s=2, t=1$ in Theorem 2.1 and then using L'Hospital rule twice for $x=-1$ we obtain the following theorem).

Theorem 3.18. If $r=0, s=2, t=1$ then for $n \geq 0$ we have the following formulas:

(a): $\sum_{k=0}^{n} k(-1)^{k} W_{k}^{2}=\frac{1}{100}\left((-1)^{n}\left(\left(20 n^{2}-30 n-1569\right) W_{n+3}^{2}-\left(20 n^{2}-70 n-1519\right) W_{n+2}^{2}-\left(20 n^{2}-90 n-\right.\right.\right.$ $\left.1679) W_{n+1}^{2}+4\left(5 n^{2}-401\right) W_{n+3} W_{n+2}-4\left(5 n^{2}+10 n-396\right) W_{n+3} W_{n+1}-4\left(15 n^{2}-10 n-1198\right) W_{n+2} W_{n+1}\right)-$ $\left.1519 W_{2}^{2}+1429 W_{1}^{2}+1569 W_{0}^{2}-1584 W_{2} W_{1}+1604 W_{2} W_{0}+4692 W_{1} W_{0}\right)$.

(b): $\sum_{k=0}^{n} k(-1)^{k} W_{k+1} W_{k}=\frac{1}{100}\left((-1)^{n}\left(2\left(5 n^{2}-401\right) W_{n+3}^{2}-2\left(5 n^{2}-10 n-396\right) W_{n+2}^{2}-2\left(5 n^{2}+10 n-\right.\right.\right.$ $\left.396) W_{n+1}^{2}+\left(10 n^{2}-10 n-827\right) W_{n+3} W_{n+2}-\left(10 n^{2}+10 n-827\right) W_{n+3} W_{n+1}-\left(30 n^{2}-50 n-2461\right) W_{n+2} W_{n+1}\right)-$ $\left.792 W_{2}^{2}+762 W_{1}^{2}+802 W_{0}^{2}-807 W_{2} W_{1}+827 W_{2} W_{0}+2381 W_{1} W_{0}\right)$.

(c): $\sum_{k=0}^{n} k(-1)^{k} W_{k+2} W_{k}=\frac{1}{100}\left((-1)^{n}\left(2\left(15 n^{2}-40 n-1173\right) W_{n+3}^{2}-2\left(15 n^{2}-70 n-1118\right) W_{n+2}^{2}-2\left(15 n^{2}-\right.\right.\right.$ $10 n-1198) W_{n+1}^{2}+\left(30 n^{2}-10 n-2381\right) W_{n+3} W_{n+2}-\left(30 n^{2}-50 n-2461\right) W_{n+3} W_{n+1}-\left(90 n^{2}-90 n-7103\right)$ $\left.\left.W_{n+2} W_{n+1}\right)-2236 W_{2}^{2}+2066 W_{1}^{2}+2346 W_{0}^{2}-2341 W_{2} W_{1}+2381 W_{2} W_{0}+6923 W_{1} W_{0}\right)$.

From Theorem 3.18, we have the following corollary which gives sum formulas of Pell-Padovan numbers (take $W_{n}=R_{n}$ with $\left.Q_{0}=1, R_{1}=1, R_{2}=1\right)$

Corollary 3.19. For $n \geq 0$, Pell-Padovan numbers have the following properties:

(a): $\sum_{k=0}^{n} k(-1)^{k} R_{k}^{2}=\frac{1}{100}\left((-1)^{n}\left(\left(20 n^{2}-30 n-1569\right) R_{n+3}^{2}-\left(20 n^{2}-70 n-1519\right) R_{n+2}^{2}-\left(20 n^{2}-90 n-\right.\right.\right.$ $\left.\left.1679) R_{n+1}^{2}+4\left(5 n^{2}-401\right) R_{n+3} R_{n+2}-4\left(5 n^{2}+10 n-396\right) R_{n+3} R_{n+1}-4\left(15 n^{2}-10 n-1198\right) R_{n+2} R_{n+1}\right)+6191\right)$. 
(b): $\sum_{k=0}^{n} k(-1)^{k} R_{k+1} R_{k}=\frac{1}{100}\left((-1)^{n}\left(2\left(5 n^{2}-401\right) R_{n+3}^{2}-2\left(5 n^{2}-10 n-396\right) R_{n+2}^{2}-2\left(5 n^{2}+10 n-396\right) R_{n+1}^{2}+\right.\right.$ $\left.\left.\left(10 n^{2}-10 n-827\right) R_{n+3} R_{n+2}-\left(10 n^{2}+10 n-827\right) R_{n+3} R_{n+1}-\left(30 n^{2}-50 n-2461\right) R_{n+2} R_{n+1}\right)+3173\right)$.

(c): $\sum_{k=0}^{n} k(-1)^{k} R_{k+2} R_{k}=\frac{1}{100}\left((-1)^{n}\left(2\left(15 n^{2}-40 n-1173\right) R_{n+3}^{2}-2\left(15 n^{2}-70 n-1118\right) R_{n+2}^{2}-2\left(15 n^{2}-\right.\right.\right.$ $10 n-1198) R_{n+1}^{2}+\left(30 n^{2}-10 n-2381\right) R_{n+3} R_{n+2}-\left(30 n^{2}-50 n-2461\right) R_{n+3} R_{n+1}-\left(90 n^{2}-90 n-7103\right)$ $\left.\left.R_{n+2} R_{n+1}\right)+9139\right)$.

Taking $W_{n}=C_{n}$ with $C_{0}=3, C_{1}=0, C_{2}=2$ in Theorem 3.18, we have the following corollary which presents sum formulas of Pell-Perrin numbers.

Corollary 3.20. For $n \geq 0$, Pell-Perrin numbers have the following properties:

(a): $\sum_{k=0}^{n} k(-1)^{k} C_{k}^{2}=\frac{1}{100}\left((-1)^{n}\left(\left(20 n^{2}-30 n-1569\right) C_{n+3}^{2}-\left(20 n^{2}-70 n-1519\right) C_{n+2}^{2}-\left(20 n^{2}-90 n-\right.\right.\right.$ 1679) $\left.\left.C_{n+1}^{2}+4\left(5 n^{2}-401\right) C_{n+3} C_{n+2}-4\left(5 n^{2}+10 n-396\right) C_{n+3} C_{n+1}-4\left(15 n^{2}-10 n-1198\right) C_{n+2} C_{n+1}\right)+17669\right)$.

(b): $\sum_{k=0}^{n} k(-1)^{k} C_{k+1} C_{k}=\frac{1}{100}\left((-1)^{n}\left(2\left(5 n^{2}-401\right) C_{n+3}^{2}-2\left(5 n^{2}-10 n-396\right) C_{n+2}^{2}-2\left(5 n^{2}+10 n-396\right) C_{n+1}^{2}+\right.\right.$ $\left.\left.\left(10 n^{2}-10 n-827\right) C_{n+3} C_{n+2}-\left(10 n^{2}+10 n-827\right) C_{n+3} C_{n+1}-\left(30 n^{2}-50 n-2461\right) C_{n+2} C_{n+1}\right)+9012\right)$.

(c): $\sum_{k=0}^{n} k(-1)^{k} C_{k+2} C_{k}=\frac{1}{100}\left((-1)^{n}\left(2\left(15 n^{2}-40 n-1173\right) C_{n+3}^{2}-2\left(15 n^{2}-70 n-1118\right) C_{n+2}^{2}-2\left(15 n^{2}-\right.\right.\right.$ $10 n-1198) C_{n+1}^{2}+\left(30 n^{2}-10 n-2381\right) C_{n+3} C_{n+2}-\left(30 n^{2}-50 n-2461\right) C_{n+3} C_{n+1}-\left(90 n^{2}-90 n-7103\right)$ $\left.\left.C_{n+2} C_{n+1}\right)+26456\right)$.

Taking $x=-1, r=0, s=1, t=2$ in Theorem 2.1, we obtain the following proposition.

Proposition 3.21. If $r=0, s=1, t=2$ then for $n \geq 0$ we have the following formulas:

(a): $\sum_{k=0}^{n} k(-1)^{k} W_{k}^{2}=\frac{1}{64}\left((-1)^{n}\left((12 n+5) W_{n+3}^{2}-(12 n-7) W_{n+2}^{2}+(16 n-4) W_{n+1}^{2}+2(4 n+1) W_{n+3} W_{n+2}-4\right.\right.$ $\left.\left.(4 n+5) W_{n+3} W_{n+1}-4(4 n-1) W_{n+2} W_{n+1}\right)-7 W_{2}^{2}+19 W_{1}^{2}-20 W_{0}^{2}-6 W_{2} W_{1}-4 W_{2} W_{0}+20 W_{1} W_{0}\right)$.

(b): $\sum_{k=0}^{n} k(-1)^{k} W_{k+1} W_{k}=\frac{1}{64}\left((-1)^{n}\left((4 n+1) W_{n+3}^{2}-(4 n-3) W_{n+2}^{2}-4(4 n+5) W_{n+1}^{2}-(8 n-2) W_{n+3} W_{n+2}+\right.\right.$ $\left.\left.2(8 n+6) W_{n+3} W_{n+1}+(16 n-12) W_{n+2} W_{n+1}\right)-3 W_{2}^{2}+7 W_{1}^{2}-4 W_{0}^{2}+10 W_{2} W_{1}-4 W_{2} W_{0}-28 W_{1} W_{0}\right)$.

(c): $\sum_{k=0}^{n} k(-1)^{k} W_{k+2} W_{k}=\frac{1}{64}\left((-1)^{n}\left((4 n-5) W_{n+3}^{2}-(4 n-9) W_{n+2}^{2}-4(4 n-1) W_{n+1}^{2}+(24 n+14) W_{n+3} W_{n+2}+\right.\right.$ $\left.\left.(16 n-12) W_{n+3} W_{n+1}-2(24 n+2) W_{n+2} W_{n+1}\right)-9 W_{2}^{2}+13 W_{1}^{2}+20 W_{0}^{2}-10 W_{2} W_{1}-28 W_{2} W_{0}+44 W_{1} W_{0}\right)$.

From Proposition 3.21, we have the following Corollary which gives sum formulas of Jacobsthal-Padovan numbers (take $W_{n}=Q_{n}$ with $\left.Q_{0}=1, Q_{1}=1, Q_{2}=1\right)$.

Corollary 3.22. For $n \geq 0$, Jacobsthal-Padovan numbers have the following properties:

(a): $\sum_{k=0}^{n} k(-1)^{k} Q_{k}^{2}=\frac{1}{64}\left((-1)^{n}\left((12 n+5) Q_{n+3}^{2}-(12 n-7) Q_{n+2}^{2}+(16 n-4) Q_{n+1}^{2}+2(4 n+1) Q_{n+3} Q_{n+2}-4\right.\right.$ $\left.\left.(4 n+5) Q_{n+3} Q_{n+1}-4(4 n-1) Q_{n+2} Q_{n+1}\right)+2\right)$

(b): $\sum_{k=0}^{n} k(-1)^{k} Q_{k+1} Q_{k}=\frac{1}{64}\left((-1)^{n}\left((4 n+1) Q_{n+3}^{2}-(4 n-3) Q_{n+2}^{2}-4(4 n+5) Q_{n+1}^{2}-(8 n-2) Q_{n+3} Q_{n+2}+2\right.\right.$ $\left.\left.(8 n+6) Q_{n+3} Q_{n+1}+(16 n-12) Q_{n+2} Q_{n+1}\right)-22\right)$.

(c): $\sum_{k=0}^{n} k(-1)^{k} Q_{k+2} Q_{k}=\frac{1}{64}\left((-1)^{n}\left((4 n-5) Q_{n+3}^{2}-(4 n-9) Q_{n+2}^{2}-4(4 n-1) Q_{n+1}^{2}+(24 n+14) Q_{n+3} Q_{n+2}+\right.\right.$ $\left.\left.(16 n-12) Q_{n+3} Q_{n+1}-2(24 n+2) Q_{n+2} Q_{n+1}\right)+30\right)$.

Taking $W_{n}=L_{n}$ with $L_{0}=3, L_{1}=0, L_{2}=2$ in Proposition 3.21, we have the following Corollary which presents sum formulas of Jacobsthal-Perrin numbers.

Corollary 3.23. For $n \geq 0$, Jacobsthal-Perrin numbers have the following properties: 
(a): $\sum_{k=0}^{n} k(-1)^{k} L_{k}^{2}=\frac{1}{64}\left((-1)^{n}\left((12 n+5) L_{n+3}^{2}-(12 n-7) L_{n+2}^{2}+(16 n-4) L_{n+1}^{2}+2(4 n+1) L_{n+3} L_{n+2}-4\right.\right.$ $\left.\left.(4 n+5) L_{n+3} L_{n+1}-4(4 n-1) L_{n+2} L_{n+1}\right)-232\right)$.

(b): $\sum_{k=0}^{n} k(-1)^{k} L_{k+1} L_{k}=\frac{1}{64}\left((-1)^{n}\left((4 n+1) L_{n+3}^{2}-(4 n-3) L_{n+2}^{2}-4(4 n+5) L_{n+1}^{2}-(8 n-2) L_{n+3} L_{n+2}+2\right.\right.$ $\left.\left.(8 n+6) L_{n+3} L_{n+1}+(16 n-12) L_{n+2} L_{n+1}\right)-72\right)$.

(c): $\sum_{k=0}^{n} k(-1)^{k} L_{k+2} L_{k}=\frac{1}{64}\left((-1)^{n}\left((4 n-5) L_{n+3}^{2}-(4 n-9) L_{n+2}^{2}-4(4 n-1) L_{n+1}^{2}+(24 n+14) L_{n+3} L_{n+2}+\right.\right.$ $\left.\left.(16 n-12) L_{n+3} L_{n+1}-2(24 n+2) L_{n+2} L_{n+1}\right)-24\right)$.

Taking $x=-1, r=1, s=0, t=1$ in Theorem 2.1, we obtain the following Proposition.

Proposition 3.24. If $r=1, s=0, t=1$ then for $n \geq 0$ we have the following formulas:

(a): $\sum_{k=0}^{n} k(-1)^{k} W_{k}^{2}=\frac{1}{9}\left((-1)^{n}\left((3 n+7) W_{n+3}^{2}-(6 n+5) W_{n+2}^{2}+(6 n-1) W_{n+1}^{2}-6 W_{n+3} W_{n+2}-2(3 n+7)\right.\right.$ $\left.\left.W_{n+3} W_{n+1}+2(3 n+10) W_{n+2} W_{n+1}\right)+4 W_{2}^{2}+W_{1}^{2}-7 W_{0}^{2}-6 W_{2} W_{1}-8 W_{2} W_{0}+14 W_{1} W_{0}\right)$.

(b): $\sum_{k=0}^{n} k(-1)^{k} W_{k+1} W_{k}=\frac{1}{9}\left((-1)^{n}\left((3 n+4) W_{n+3}^{2}+(3 n+10) W_{n+2}^{2}-(3 n+7) W_{n+1}^{2}-(9 n+15) W_{n+3} W_{n+2}+\right.\right.$ $\left.\left.(3 n+10) W_{n+3} W_{n+1}+(6 n-4) W_{n+2} W_{n+1}\right)+W_{2}^{2}+7 W_{1}^{2}-4 W_{0}^{2}-6 W_{2} W_{1}+7 W_{2} W_{0}-10 W_{1} W_{0}\right)$.

(c): $\sum_{k=0}^{n} k(-1)^{k} W_{k+2} W_{k}=\frac{1}{9}\left((-1)^{n}\left(-3 W_{n+3}^{2}-3 W_{n+2}^{2}+3 W_{n+1}^{2}+9 W_{n+3} W_{n+2}+(9 n+6) W_{n+3} W_{n+1}-\right.\right.$ $\left.\left.(9 n+15) W_{n+2} W_{n+1}\right)-3 W_{2}^{2}-3 W_{1}^{2}+3 W_{0}^{2}+9 W_{2} W_{1}-3 W_{2} W_{0}-6 W_{1} W_{0}\right)$.

From Proposition 3.24, we have the following corollary which gives sum formulas of Narayana numbers (take $W_{n}=N_{n}$ with $\left.N_{0}=0, N_{1}=1, N_{2}=1\right)$.

Corollary 3.25. For $n \geq 0$, Narayana numbers have the following properties:

(a): $\sum_{k=0}^{n} k(-1)^{k} N_{k}^{2}=\frac{1}{9}\left((-1)^{n}\left((3 n+7) N_{n+3}^{2}-(6 n+5) N_{n+2}^{2}+(6 n-1) N_{n+1}^{2}-6 N_{n+3} N_{n+2}-2(3 n+7)\right.\right.$ $\left.\left.N_{n+3} N_{n+1}+2(3 n+10) N_{n+2} N_{n+1}\right)-1\right)$.

(b): $\sum_{k=0}^{n} k(-1)^{k} N_{k+1} N_{k}=\frac{1}{9}\left((-1)^{n}\left((3 n+4) N_{n+3}^{2}+(3 n+10) N_{n+2}^{2}-(3 n+7) N_{n+1}^{2}-(9 n+15) N_{n+3} N_{n+2}+\right.\right.$ $\left.\left.(3 n+10) N_{n+3} N_{n+1}+(6 n-4) N_{n+2} N_{n+1}\right)+2\right)$.

(c): $\sum_{k=0}^{n} k(-1)^{k} N_{k+2} N_{k}=\frac{1}{9}\left((-1)^{n}\left(-3 N_{n+3}^{2}-3 N_{n+2}^{2}+3 N_{n+1}^{2}+9 N_{n+3} N_{n+2}+(9 n+6) N_{n+3} N_{n+1}-(9 n+15)\right.\right.$ $\left.\left.N_{n+2} N_{n+1}\right)+3\right)$.

Taking $W_{n}=U_{n}$ with $U_{0}=3, U_{1}=1, U_{2}=1$ in Proposition 3.24 , we have the following corollary which presents sum formulas of Narayana-Lucas numbers.

Corollary 3.26. For $n \geq 0$, Narayana-Lucas numbers have the following properties:

(a): $\sum_{k=0}^{n} k(-1)^{k} U_{k}^{2}=\frac{1}{9}\left((-1)^{n}\left((3 n+7) U_{n+3}^{2}-(6 n+5) U_{n+2}^{2}+(6 n-1) U_{n+1}^{2}-6 U_{n+3} U_{n+2}-2(3 n+7)\right.\right.$ $\left.\left.U_{n+3} U_{n+1}+2(3 n+10) U_{n+2} U_{n+1}\right)-46\right)$.

(b): $\sum_{k=0}^{n} k(-1)^{k} U_{k+1} U_{k}=\frac{1}{9}\left((-1)^{n}\left((3 n+4) U_{n+3}^{2}+(3 n+10) U_{n+2}^{2}-(3 n+7) U_{n+1}^{2}-(9 n+15) U_{n+3} U_{n+2}+\right.\right.$ $\left.\left.(3 n+10) U_{n+3} U_{n+1}+(6 n-4) U_{n+2} U_{n+1}\right)-43\right)$.

(c): $\sum_{k=0}^{n} k(-1)^{k} U_{k+2} U_{k}=\frac{1}{9}\left((-1)^{n}\left(-3 U_{n+3}^{2}-3 U_{n+2}^{2}+3 U_{n+1}^{2}+9 U_{n+3} U_{n+2}+(9 n+6) U_{n+3} U_{n+1}-(9 n+15)\right.\right.$ $\left.\left.U_{n+2} U_{n+1}\right)+3\right)$.

From Proposition 3.24, we have the following corollary which gives sum formulas of Narayana-Perrin numbers (take $W_{n}=H_{n}$ with $H_{0}=3, H_{1}=0, H_{2}=2$ ).

COROllary 3.27. For $n \geq 0$, Narayana-Perrin numbers have the following properties: 
(a): $\sum_{k=0}^{n} k(-1)^{k} H_{k}^{2}=\frac{1}{9}\left((-1)^{n}\left((3 n+7) H_{n+3}^{2}-(6 n+5) H_{n+2}^{2}+(6 n-1) H_{n+1}^{2}-6 H_{n+3} H_{n+2}-2(3 n+7)\right.\right.$ $\left.\left.H_{n+3} H_{n+1}+2(3 n+10) H_{n+2} H_{n+1}\right)-95\right)$.

(b): $\sum_{k=0}^{n} k(-1)^{k} H_{k+1} H_{k}=\frac{1}{9}\left((-1)^{n}\left((3 n+4) H_{n+3}^{2}+(3 n+10) H_{n+2}^{2}-(3 n+7) H_{n+1}^{2}-(9 n+15) H_{n+3} H_{n+2}+\right.\right.$ $\left.\left.(3 n+10) H_{n+3} H_{n+1}+(6 n-4) H_{n+2} H_{n+1}\right)+10\right)$.

(c): $\sum_{k=0}^{n} k(-1)^{k} H_{k+2} H_{k}=\frac{1}{9}\left((-1)^{n}\left(-3 H_{n+3}^{2}-3 H_{n+2}^{2}+3 H_{n+1}^{2}+9 H_{n+3} H_{n+2}+(9 n+6) H_{n+3} H_{n+1}-(9 n+15)\right.\right.$ $\left.\left.H_{n+2} H_{n+1}\right)-3\right)$.

Taking $x=-1, r=1, s=1, t=2$ in Theorem 2.1, we obtain the following proposition.

Proposition 3.28. If $r=1, s=1, t=2$ then for $n \geq 0$ we have the following formulas:

(a): $\sum_{k=0}^{n} k(-1)^{k} W_{k}^{2}=\frac{1}{150}\left((-1)^{n}\left((20 n+19) W_{n+3}^{2}-(30 n-19) W_{n+2}^{2}+(70 n-6) W_{n+1}^{2}-(10 n+37) W_{n+3} W_{n+2}-\right.\right.$ $\left.\left.2(30 n+31) W_{n+3} W_{n+1}+4(10 n+22) W_{n+2} W_{n+1}\right)-W_{2}^{2}+49 W_{1}^{2}-76 W_{0}^{2}-27 W_{2} W_{1}-2 W_{2} W_{0}+48 W_{1} W_{0}\right)$.

(b): $\sum_{k=0}^{n} k(-1)^{k} W_{k+1} W_{k}=\frac{1}{300}\left((-1)^{n}\left((30 n+1) W_{n+3}^{2}+(30 n+51) W_{n+2}^{2}-4(30 n+31) W_{n+1}^{2}-(90 n+\right.\right.$ 23) $\left.W_{n+3} W_{n+2}+2(30 n+51) W_{n+3} W_{n+1}+(60 n-148) W_{n+2} W_{n+1}\right)-29 W_{2}^{2}+21 W_{1}^{2}-4 W_{0}^{2}+67 W_{2} W_{1}+$ $\left.42 W_{2} W_{0}-208 W_{1} W_{0}\right)$.

(c): $\sum_{k=0}^{n} k(-1)^{k} W_{k+2} W_{k}=\frac{1}{900}\left((-1)^{n}\left((30 n-69) W_{n+3}^{2}-(270 n+219) W_{n+2}^{2}-4(30 n-39) W_{n+1}^{2}+(210 n+\right.\right.$ $\left.387) W_{n+3} W_{n+2}+(360 n-138) W_{n+3} W_{n+1}-2(420 n+144) W_{n+2} W_{n+1}\right)-99 W_{2}^{2}+51 W_{1}^{2}+276 W_{0}^{2}+177$ $\left.W_{2} W_{1}-498 W_{2} W_{0}+552 W_{1} W_{0}\right)$.

From Proposition 3.28, we have the following corollary which gives sum formulas of third order Jacobsthal numbers (take $W_{n}=J_{n}$ with $J_{0}=0, J_{1}=1, J_{2}=1$ ).

CoRollary 3.29. For $n \geq 0$, third order Jacobsthal numbers have the following properties:

(a): $\sum_{k=0}^{n} k(-1)^{k} J_{k}^{2}=\frac{1}{150}\left((-1)^{n}\left((20 n+19) J_{n+3}^{2}-(30 n-19) J_{n+2}^{2}+(70 n-6) J_{n+1}^{2}-(10 n+37) J_{n+3} J_{n+2}-2\right.\right.$ $\left.\left.(30 n+31) J_{n+3} J_{n+1}+4(10 n+22) J_{n+2} J_{n+1}\right)+21\right)$.

(b): $\sum_{k=0}^{n} k(-1)^{k} J_{k+1} J_{k}=\frac{1}{300}\left((-1)^{n}\left((30 n+1) J_{n+3}^{2}+(30 n+51) J_{n+2}^{2}-4(30 n+31) J_{n+1}^{2}-(90 n+23) J_{n+3} J_{n+2}+\right.\right.$ $\left.\left.2(30 n+51) J_{n+3} J_{n+1}+(60 n-148) J_{n+2} J_{n+1}\right)+59\right)$.

(c): $\sum_{k=0}^{n} k(-1)^{k} J_{k+2} J_{k}=\frac{1}{900}\left((-1)^{n}\left((30 n-69) J_{n+3}^{2}-(270 n+219) J_{n+2}^{2}-4(30 n-39) J_{n+1}^{2}+(210 n+\right.\right.$ $\left.\left.387) J_{n+3} J_{n+2}+(360 n-138) J_{n+3} J_{n+1}-2(420 n+144) J_{n+2} J_{n+1}\right)+129\right)$.

Taking $W_{n}=j_{n}$ with $j_{0}=2, j_{1}=1, j_{2}=5$ in Proposition 3.28 , we have the following corollary which presents sum formulas of third order Jacobsthal-Lucas numbers.

Corollary 3.30. For $n \geq 0$, third order Jacobsthal-Lucas numbers have the following properties:

(a): $\sum_{k=0}^{n} k(-1)^{k} j_{k}^{2}=\frac{1}{150}\left((-1)^{n}\left((20 n+19) j_{n+3}^{2}-(30 n-19) j_{n+2}^{2}+(70 n-6) j_{n+1}^{2}-(10 n+37) j_{n+3} j_{n+2}-2\right.\right.$ $\left.\left.(30 n+31) j_{n+3} j_{n+1}+4(10 n+22) j_{n+2} j_{n+1}\right)-339\right)$.

(b): $\sum_{k=0}^{n} k(-1)^{k} j_{k+1} j_{k}=\frac{1}{300}\left((-1)^{n}\left((30 n+1) j_{n+3}^{2}+(30 n+51) j_{n+2}^{2}-4(30 n+31) j_{n+1}^{2}-(90 n+23) j_{n+3} j_{n+2}+\right.\right.$ $\left.\left.2(30 n+51) j_{n+3} j_{n+1}+(60 n-148) j_{n+2} j_{n+1}\right)-381\right)$.

(c): $\sum_{k=0}^{n} k(-1)^{k} j_{k+2} j_{k}=\frac{1}{900}\left((-1)^{n}\left((30 n-69) j_{n+3}^{2}-(270 n+219) j_{n+2}^{2}-4(30 n-39) j_{n+1}^{2}+(210 n+\right.\right.$ $\left.\left.387) j_{n+3} j_{n+2}+(360 n-138) j_{n+3} j_{n+1}-2(420 n+144) j_{n+2} j_{n+1}\right)-4311\right)$.

Taking $x=-1, r=2, s=3, t=5$ in Theorem 2.1, we obtain the following Proposition. 
Proposition 3.31. If $r=2, s=3, t=5$ then for $n \geq 0$ we have the following formulas:

(a): $\sum_{k=0}^{n} k(-1)^{k} W_{k}^{2}=\frac{1}{680625}\left((-1)^{n}\left((15675 n+11674) W_{n+3}^{2}-(9075 n-81169) W_{n+2}^{2}+(288750 n-3100) W_{n+1}^{2}-\right.\right.$ $\left.2(17325 n+30966) W_{n+3} W_{n+2}-10(14025 n+6407) W_{n+3} W_{n+1}+10(23100 n+20113) W_{n+2} W_{n+1}\right)-4001 W_{2}^{2}+$ $\left.90244 W_{1}^{2}-291850 W_{0}^{2}-27282 W_{2} W_{1}+76180 W_{2} W_{0}-29870 W_{1} W_{0}\right)$.

(b): $\sum_{k=0}^{n} k(-1)^{k} W_{k+1} W_{k}=\frac{1}{680625}\left((-1)^{n}\left((14025 n-7618) W_{n+3}^{2}+11(5775 n+697) W_{n+2}^{2}-25(14025 n+\right.\right.$ $\left.6407) W_{n+1}^{2}-(66825 n-22224) W_{n+3} W_{n+2}+5(10725 n+18973) W_{n+3} W_{n+1}-(8250 n+254035) W_{n+2} W_{n+1}\right)-$ $\left.21643 W_{2}^{2}-55858 W_{1}^{2}+190450 W_{0}^{2}+89049 W_{2} W_{1}+41240 W_{2} W_{0}-245785 W_{1} W_{0}\right)$.

(c): $\sum_{k=0}^{n} k(-1)^{k} W_{k+2} W_{k}=\frac{1}{75625}\left((-1)^{n}(550 n-1361) W_{n+3}^{2}-11(-1)^{n}(2200 n+981) W_{n+2}^{2}-25(-1)^{n}(550 n-\right.$ $811) W_{n+1}^{2}-5(-1)^{n}(14300 n-1011) W_{n+2} W_{n+1}+5(-1)^{n}(2200 n-1429) W_{n+3} W_{n+1}+(-1)^{n}(10725 n+$ $\left.9073) W_{n+3} W_{n+2}-1911 W_{2}^{2}+13409 W_{1}^{2}+34025 W_{0}^{2}-1652 W_{2} W_{1}-18145 W_{2} W_{0}+76555 W_{1} W_{0}\right)$.

From Proposition 3.31, we have the following corollary which gives sum formulas of 3-primes numbers (take $W_{n}=G_{n}$ with $\left.G_{0}=0, G_{1}=1, G_{2}=2\right)$.

Corollary 3.32. For $n \geq 0$, 3-primes numbers have the following properties:

(a): $\sum_{k=0}^{n} k(-1)^{k} G_{k}^{2}=\frac{1}{680625}\left((-1)^{n}\left((15675 n+11674) G_{n+3}^{2}-(9075 n-81169) G_{n+2}^{2}+(288750 n-3100) G_{n+1}^{2}-\right.\right.$ $\left.\left.2(17325 n+30966) G_{n+3} G_{n+2}-10(14025 n+6407) G_{n+3} G_{n+1}+10(23100 n+20113) G_{n+2} G_{n+1}\right)+19676\right)$.

(b): $\sum_{k=0}^{n} k(-1)^{k} G_{k+1} G_{k}=\frac{1}{680625}\left((-1)^{n}\left((14025 n-7618) G_{n+3}^{2}+11(5775 n+697) G_{n+2}^{2}-25(14025 n+\right.\right.$ $\left.6407) G_{n+1}^{2}-(66825 n-22224) G_{n+3} G_{n+2}+5(10725 n+18973) G_{n+3} G_{n+1}-(8250 n+254035) G_{n+2} G_{n+1}\right)+$ 35668).

(c): $\sum_{k=0}^{n} k(-1)^{k} G_{k+2} G_{k}=\frac{1}{75625}\left((-1)^{n}(550 n-1361) G_{n+3}^{2}-11(-1)^{n}(2200 n+981) G_{n+2}^{2}-25(-1)^{n}(550 n-\right.$ $811) G_{n+1}^{2}-5(-1)^{n}(14300 n-1011) G_{n+2} G_{n+1}+5(-1)^{n}(2200 n-1429) G_{n+3} G_{n+1}+(-1)^{n}(10725 n+$ 9073) $\left.G_{n+3} G_{n+2}+2461\right)$.

Taking $W_{n}=H_{n}$ with $H_{0}=3, H_{1}=2, H_{2}=10$ in Proposition 3.31, we have the following corollary which presents sum formulas of Lucas 3-primes numbers.

Corollary 3.33. For $n \geq 0$, Lucas 3-primes numbers have the following properties:

(a): $\sum_{k=0}^{n} k(-1)^{k} H_{k}^{2}=\frac{1}{680625}\left((-1)^{n}\left((15675 n+11674) H_{n+3}^{2}-(9075 n-81169) H_{n+2}^{2}+(288750 n-3100) H_{n+1}^{2}-\right.\right.$ $\left.\left.2(17325 n+30966) H_{n+3} H_{n+2}-10(14025 n+6407) H_{n+3} H_{n+1}+10(23100 n+20113) H_{n+2} H_{n+1}\right)-1105234\right)$.

(b): $\sum_{k=0}^{n} k(-1)^{k} H_{k+1} H_{k}=\frac{1}{680625}\left((-1)^{n}\left((14025 n-7618) H_{n+3}^{2}+11(5775 n+697) H_{n+2}^{2}-25(14025 n+\right.\right.$ $\left.6407) H_{n+1}^{2}-(66825 n-22224) H_{n+3} H_{n+2}+5(10725 n+18973) H_{n+3} H_{n+1}-(8250 n+254035) H_{n+2} H_{n+1}\right)+$ 869788).

(c): $\sum_{k=0}^{n} k(-1)^{k} H_{k+2} H_{k}=\frac{1}{75625}\left((-1)^{n}(550 n-1361) H_{n+3}^{2}-11(-1)^{n}(2200 n+981) H_{n+2}^{2}-25(-1)^{n}(550 n-\right.$ 811) $H_{n+1}^{2}-5(-1)^{n}(14300 n-1011) H_{n+2} H_{n+1}+5(-1)^{n}(2200 n-1429) H_{n+3} H_{n+1}+(-1)^{n}(10725 n+$ 9073) $\left.H_{n+3} H_{n+2}+50701\right)$.

From Proposition 3.31, we have the following corollary which gives sum formulas of modified 3-primes numbers (take $W_{n}=E_{n}$ with $\left.E_{0}=0, E_{1}=1, E_{2}=1\right)$.

Corollary 3.34. For $n \geq 0$, modified 3-primes numbers have the following properties: 
(a): $\sum_{k=0}^{n} k(-1)^{k} E_{k}^{2}=\frac{1}{680625}\left((-1)^{n}\left((15675 n+11674) E_{n+3}^{2}-(9075 n-81169) E_{n+2}^{2}+(288750 n-3100) E_{n+1}^{2}-\right.\right.$ $\left.\left.2(17325 n+30966) E_{n+3} E_{n+2}-10(14025 n+6407) E_{n+3} E_{n+1}+10(23100 n+20113) E_{n+2} E_{n+1}\right)+58961\right)$.

(b): $\sum_{k=0}^{n} k(-1)^{k} E_{k+1} E_{k}=\frac{1}{680625}\left((-1)^{n}\left((14025 n-7618) E_{n+3}^{2}+11(5775 n+697) E_{n+2}^{2}-25(14025 n+6407) E_{n+1}^{2}-\right.\right.$ $\left.\left.(66825 n-22224) E_{n+3} E_{n+2}+5(10725 n+18973) E_{n+3} E_{n+1}-(8250 n+254035) E_{n+2} E_{n+1}\right)+11548\right)$.

(c): $\sum_{k=0}^{n} k(-1)^{k} E_{k+2} E_{k}=\frac{1}{75625}\left((-1)^{n}(550 n-1361) E_{n+3}^{2}-11(-1)^{n}(2200 n+981) E_{n+2}^{2}-25(-1)^{n}(550 n-\right.$ $811) E_{n+1}^{2}-5(-1)^{n}(14300 n-1011) E_{n+2} E_{n+1}+5(-1)^{n}(2200 n-1429) E_{n+3} E_{n+1}+(-1)^{n}(10725 n+$ 9073) $\left.E_{n+3} E_{n+2}+9846\right)$.

3.3. The Case $x=i$. We now consider the complex case $x=i$ in Theorem 2.1. The following proposition presents some summing formulas of generalized Fibonacci numbers with positive subscripts.

Taking $x=i, r=s=t=1$ in Theorem 2.1, we obtain the following Proposition.

Proposition 3.35. If $r=s=t=1$ then for $n \geq 0$ we have the following formulas:

(a): $\sum_{k=0}^{n} k i^{k} W_{k}^{2}=\frac{-i}{4}\left(i^{n}\left(i((1+i) n+2+i) W_{n+3}^{2}+(2 i n-2+4 i) W_{n+2}^{2}-i((1+3 i) n+2+i) W_{n+1}^{2}+2\right.\right.$ $\left.((1-i) n+2-3 i) W_{n+3} W_{n+2}+(2+2 i) W_{n+3} W_{n+1}-2((1+i) n+4+i) W_{n+2} W_{n+1}\right)-W_{2}^{2}-(2+2 i) W_{1}^{2}+$ $\left.(1-2 i) W_{0}^{2}+(4+2 i) W_{2} W_{1}-6 i W_{1} W_{0}-(2-2 i) W_{2} W_{0}\right)$.

(b): $\sum_{k=0}^{n} k i^{k} W_{k+1} W_{k}=\frac{-i}{4}\left(i^{n}\left((1-i) W_{n+3}^{2}-2(i n+1+2 i) W_{n+2}^{2}+(1+i) W_{n+1}^{2}+(2 i n+4 i) W_{n+3} W_{n+2}+\right.\right.$ $\left.i(2 i n+2+4 i) W_{n+3} W_{n+1}-i((2+4 i) n+4+4 i) W_{n+2} W_{n+1}\right)+(1+i) W_{2}^{2}+(2-2 i) W_{1}^{2}-(1-i) W_{0}^{2}-$ $\left.2 W_{2} W_{1}-(2+2 i) W_{2} W_{0}+2 W_{1} W_{0}\right)$.

(c): $\sum_{k=0}^{n} k i^{k} W_{k+2} W_{k}=\frac{-i}{4}\left(i^{n}\left(i((1+i) n+2-i) W_{n+3}^{2}+(2 n+2-2 i) W_{n+2}^{2}-((1+i) n+3) W_{n+1}^{2}-(2+2 i)\right.\right.$ $\left.W_{n+3} W_{n+2}-i((2+2 i) n+2) W_{n+3} W_{n+1}-(2-2 i) W_{n+2} W_{n+1}\right)-(1-2 i) W_{2}^{2}+2 W_{1}^{2}-(1+2 i) W_{0}^{2}+(2-$ $\left.2 i) W_{2} W_{1}-2 i W_{2} W_{0}-(2+2 i) W_{1} W_{0}\right)$.

From Proposition 3.35, we have the following Corollary which gives sum formulas of Tribonacci numbers (take $W_{n}=T_{n}$ with $\left.T_{0}=0, T_{1}=1, T_{2}=1\right)$.

Corollary 3.36. For $n \geq 0$, Tribonacci numbers have the following properties:

(a): $\sum_{k=0}^{n} k i^{k} T_{k}^{2}=\frac{-i}{4}\left(i^{n}\left(i((1+i) n+2+i) T_{n+3}^{2}+(2 i n-2+4 i) T_{n+2}^{2}-i((1+3 i) n+2+i) T_{n+1}^{2}+2((1-\right.\right.$ i) $\left.\left.n+2-3 i) T_{n+3} T_{n+2}+(2+2 i) T_{n+3} T_{n+1}-2((1+i) n+4+i) T_{n+2} T_{n+1}\right)+1\right)$.

(b): $\sum_{k=0}^{n} k i^{k} T_{k+1} T_{k}=\frac{-i}{4}\left(i^{n}\left((1-i) T_{n+3}^{2}-2(i n+1+2 i) T_{n+2}^{2}+(1+i) T_{n+1}^{2}+(2 i n+4 i) T_{n+3} T_{n+2}+i(2 i n+2+4 i)\right.\right.$ $\left.\left.T_{n+3} T_{n+1}-i((2+4 i) n+4+4 i) T_{n+2} T_{n+1}\right)+1-i\right)$.

(c): $\sum_{k=0}^{n} k i^{k} T_{k+2} T_{k}=\frac{-i}{4}\left(i^{n}\left(i((1+i) n+2-i) T_{n+3}^{2}+(2 n+2-2 i) T_{n+2}^{2}-((1+i) n+3) T_{n+1}^{2}-(2+2 i)\right.\right.$ $\left.\left.T_{n+3} T_{n+2}-i((2+2 i) n+2) T_{n+3} T_{n+1}-(2-2 i) T_{n+2} T_{n+1}\right)+3\right)$.

Taking $W_{n}=K_{n}$ with $K_{0}=3, K_{1}=1, K_{2}=3$ in Proposition 3.35, we have the following Corollary which presents sum formulas of Tribonacci-Lucas numbers.

Corollary 3.37. For $n \geq 0$, Tribonacci-Lucas numbers have the following properties:

(a): $\sum_{k=0}^{n} k i^{k} K_{k}^{2}=\frac{-i}{4}\left(i^{n}\left(i((1+i) n+2+i) K_{n+3}^{2}+(2 i n-2+4 i) K_{n+2}^{2}-i((1+3 i) n+2+i) K_{n+1}^{2}+2\right.\right.$ $\left.\left.((1-i) n+2-3 i) K_{n+3} K_{n+2}+(2+2 i) K_{n+3} K_{n+1}-2((1+i) n+4+i) K_{n+2} K_{n+1}\right)-8-14 i\right)$.

(b): $\sum_{k=0}^{n} k i^{k} K_{k+1} K_{k}=\frac{-i}{4}\left(i^{n}\left((1-i) K_{n+3}^{2}-2(i n+1+2 i) K_{n+2}^{2}+(1+i) K_{n+1}^{2}+(2 i n+4 i) K_{n+3} K_{n+2}+\right.\right.$ $\left.\left.i(2 i n+2+4 i) K_{n+3} K_{n+1}-i((2+4 i) n+4+4 i) K_{n+2} K_{n+1}\right)-16-2 i\right)$. 
(c): $\sum_{k=0}^{n} k i^{k} K_{k+2} K_{k}=\frac{-i}{4}\left(i^{n}\left(i((1+i) n+2-i) K_{n+3}^{2}+(2 n+2-2 i) K_{n+2}^{2}-((1+i) n+3) K_{n+1}^{2}-(2+2 i)\right.\right.$

$$
\left.\left.K_{n+3} K_{n+2}-i((2+2 i) n+2) K_{n+3} K_{n+1}-(2-2 i) K_{n+2} K_{n+1}\right)-16-30 i\right) .
$$

Corresponding sums of the other third order linear sequences can be calculated similarly when $x=i$.

\section{References}

[1] Bruce, I., A modified Tribonacci sequence, Fibonacci Quarterly, 22(3), 244-246, 1984.

[2] Catalani, M., Identities for Tribonacci-related sequences, arXiv:math/0209179, 2012.

[3] Čerin, Z., Formulae for sums of Jacobsthal-Lucas numbers, Int. Math. Forum, 2(40), 1969-1984, 2007.

[4] Čerin, Z., Sums of Squares and Products of Jacobsthal Numbers. Journal of Integer Sequences, 10, Article 07.2.5, 2007.

[5] Chen, L., Wang, X., The Power Sums Involving Fibonacci Polynomials and Their Applications, Symmetry, 11, 2019, doi.org/10.3390/sym11050635.

[6] Choi, E., Modular Tribonacci Numbers by Matrix Method, Journal of the Korean Society of Mathematical Education Series B: Pure and Applied. Mathematics. 20(3), 207-221, 2013.

[7] Elia, M., Derived Sequences, The Tribonacci Recurrence and Cubic Forms, Fibonacci Quarterly, 39 (2), $107-115,2001$.

[8] Frontczak, R.,Sums of powers of Fibonacci and Lucas numbers: A new bottom-up approach, Notes on Number Theory and Discrete Mathematics, 24(2), 94-103, 2018.

[9] Frontczak, R., Sums of Cubes Over Odd-Index Fibonacci Numbers, Integers, 18, 2018.

[10] Gnanam, A., Anitha, B., Sums of Squares Jacobsthal Numbers. IOSR Journal of Mathematics, 11(6), 62-64. 2015.

[11] Kiliç, E., Taş̧i, D., The Linear Algebra of The Pell Matrix, Boletín de la Sociedad Matemática Mexicana, 3(11), 2005.

[12] Kilıc, E., Sums of the squares of terms of sequence $\left\{u_{n}\right\}$, Proc. Indian Acad. Sci. (Math. Sci.) 118(1), 27-41, 2008.

[13] Lin, P. Y., De Moivre-Type Identities For The Tribonacci Numbers, Fibonacci Quarterly, 26, 131-134, 1988.

[14] Pethe, S., Some Identities for Tribonacci sequences, Fibonacci Quarterly, 26(2), 144-151, 1988.

[15] Prodinger, H., Sums of Powers of Fibonacci Polynomials, Proc. Indian Acad. Sci. (Math. Sci.), 119(5), 567-570, 2009.

[16] Prodinger, H., Selkirk, S.J., Sums of Squares of Tetranacci Numbers: A Generating Function Approach, 2019, http://arxiv.org/abs/1906.08336v1.

[17] Raza, Z., Riaz, M., Ali, M.A., Some Inequalities on the Norms of Special Matrices with Generalized Tribonacci and Generalized Pell-Padovan Sequences, arXiv, 2015, http://arxiv.org/abs/1407.1369v2

[18] Schumacher, R., How to sum the squares of the Tetranacci numbers and the Fibonacci m-step numbers. Fibonacci Quarterly, $57: 168-175,2019$.

[19] Scott, A., Delaney, T., Hoggatt Jr., V., The Tribonacci sequence, Fibonacci Quarterly, 15(3), $193-200,1977$.

[20] Shannon, A.G, Horadam, A.F., Some Properties of Third-Order Recurrence Relations, The Fibonacci Quarterly, 10 (2), 135-146, 1972 .

[21] Shannon, A., Tribonacci numbers and Pascal's pyramid, Fibonacci Quarterly, 15(3), pp. 268 and $275,1977$.

[22] N.J.A. Sloane, The on-line encyclopedia of integer sequences, http://oeis.org/

[23] Spickerman, W., Binet's formula for the Tribonacci sequence, Fibonacci Quarterly, 20, 118-120, 1982.

[24] Soykan, Y., Closed Formulas for the Sums of Squares of Generalized Fibonacci Numbers, Asian Journal of Advanced Research and Reports, 9(1), 23-39, 2020. https://doi.org/10.9734/ajarr/2020/v9i130212

[25] Soykan Y., Closed Formulas for the Sums of Cubes of Generalized Fibonacci Numbers: Closed Formulas of and $\sum_{k=0}^{n} W_{k}^{3}$ and $\sum_{k=1}^{n} W_{-k}^{3}$, Archives of Current Research International, 20(2), 58-69, 2020. DOI: 10.9734/ACRI/2020/v20i230177

[26] Soykan, Y., A Closed Formula for the Sums of Squares of Generalized Tribonacci numbers, Journal of Progressive Research in Mathematics, 16(2), 2932-2941, 2020.

[27] Soykan, Y., A Study On Sums of Cubes of Generalized Fibonacci Numbers: Closed Formulas of $\sum_{k=0}^{n} x^{k} W_{k}^{3}$ and $\sum_{k=1}^{n} x^{k} W_{-k}^{3}$, Preprints 2020, 2020040437 (doi: 10.20944/preprints202004.0437.v1).

[28] Soykan Y., On Sums of Cubes of Generalized Fibonacci Numbers: Closed Formulas of $\sum_{k=0}^{n} k W_{k}^{3}$ and $\sum_{k=1}^{n} k W_{-k}^{3}$, Asian Research Journal of Mathematics, 16(6), 37-52, 2020. DOI: 10.9734/ARJOM/2020/v16i630196

[29] Soykan, Y., On the Sums of Squares of Generalized Tribonacci Numbers: Closed Formulas of $\sum_{k=0}^{n} x^{k} W_{k}^{2}$, Archives of Current Research International, 20(4), 22-47, 2020. DOI: 10.9734/ACRI/2020/v20i430187

[30] Soykan, Y., Formulae For The Sums of Squares of Generalized Tribonacci Numbers: Closed Form Formulas of $\sum_{k=0}^{n} k W_{k}^{2}$, IOSR Journal of Mathematics, 16(4), 1-18, 2020. DOI: 10.9790/5728-1604010118

[31] Soykan, Y., A Study on Generalized Fibonacci Numbers: Sum Formulas $\sum_{k=0}^{n} k x^{k} W_{k}^{3}$ and $\sum_{k=1}^{n} k x^{k} W_{-k}^{3}$ for the Cubes of Terms, Earthline Journal of Mathematical Sciences, 4(2), 297-331, 2020. https://doi.org/10.34198/ejms.4220.297331

[32] Soykan Y., Generalized Fibonacci Numbers: Sum Formulas of the Squares of Terms, MathLAB Journal, Vol 5, 46-62, 2020. 
[33] Soykan, Y., Horadam Numbers: Sum of the Squares of Terms of Sequence, Int. J. Adv. Appl. Math. and Mech. In Presss.

[34] Soykan, Y., On Generalized Tetranacci Numbers: Closed Form Formulas of the Sum $\sum_{k=0}^{n} W_{k}^{2}$ of the Squares of Terms, Preprints 2020, 2020050453 (doi: 10.20944/preprints202005.0453.v1).

[35] Yalavigi, C.C., A Note on 'Another Generalized Fibonacci Sequence', The Mathematics Student. 39, 407-408, 1971.

[36] Yalavigi, C. C., Properties of Tribonacci numbers, Fibonacci Quarterly, 10(3), 231-246, 1972.

[37] Yilmaz, N., Taskara, N., Tribonacci and Tribonacci-Lucas Numbers via the Determinants of Special Matrices, Applied Mathematical Sciences, 8(39), 1947-1955, 2014.

[38] Marcellus E. Waddill, Using Matrix Techniques to Establish Properties of a Generalized Tribonacci Sequence (in Applications of Fibonacci Numbers, Volume 4, G. E. Bergum et al., eds.). Kluwer Academic Publishers. Dordrecht, The Netherlands: pp. 299-308, 1991.

[39] Wamiliana., Suharsono., Kristanto, P. E., Counting the sum of cubes for Lucas and Gibonacci Numbers, Science and Technology Indonesia, 4(2), 31-35, 2019. 